\title{
One-Shot Determination of Residual Dipolar Couplings: Application to the Structural Discrimination of Small Molecules Containing Multiple Stereocenters.
}

\author{
Laura Castañar, ${ }^{2}$ Manuela Garcia, ${ }^{1}$ Erich Hellemann, ${ }^{1}$ Pau Nolis ${ }^{2}$ \\ Roberto R. Gil, ${ }^{1, *}$ and Teodor Parella ${ }^{2, *}$ \\ ${ }^{1}$ Department of Chemistry, Carnegie Mellon University, Pittsburgh, PA, USA; \\ ${ }^{2}$ Servei de Ressonància Magnètica Nuclear, Universitat Autònoma de Barcelona, \\ Facultat de Ciències, E-08193 Bellaterra (Barcelona), Catalonia, Spain.
}




\section{Table of contents:}

- Figure S1: Pulse sequence scheme for real-time BIRD-based homodecoupled $J$ scaled BIRD HSQC (HD-JSB-HSQC) experiment ............................ 4

- Figure S2: Comparison between the A) conventional JSB-HSQC and B) HD-JSBHSQC spectra of estradiol .................................................5

- Figure S3: Comparison between the A) conventional $J$-HSQC and B) HD-J-HSQC

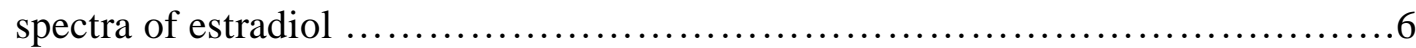

- Figure S4: Comparison between several A-D) HD-JSB-HSQC ( $J$-scaling factors of $1,2,4$ and 8 respectively) and E) the analog HD-J-HSQC spectra of estradiol .......7

- Figure S5: Effect of nonuniform sampling (NUS) in the experimental time of the

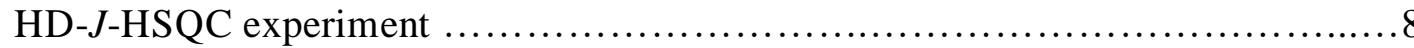

- Figure S6: Effect of non-uniform sampling (NUS) in the signal resolution of HD-J-

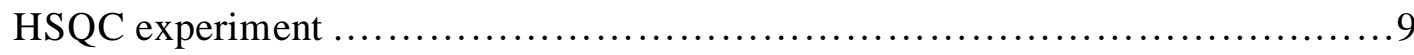

- Figure S7: a) Conventional $J$-HSQC, b) equivalent HD- $J$-HSQC with real-time homodecoupling during acquisition and C) HD-J-HSQC spectra using $25 \%$ NUS of strychnine

- Figure S8: Edited $\mathrm{CH} / \mathrm{CH}_{2}$ internal $\mathrm{F}_{1}$ projections extracted from $\mathrm{B}$ ) $J$-HSQC with INEPT, C) HD-J-HSQC with INEPT and D) HD-J-HSQC with PEP transfer spectra of strychnine

- Figure S9: (left) 2D $J$-HSQC and (right) 2D HD-J-HSQC spectra of an anisotropic sample of strychnine dissolved in PMMA/ $\mathrm{CDCl}_{3}$

- Figure S10: Expansions extracted from spectra of Fig. S9 that show the multiplet $J$ pattern simplification achieved in the HD-J-HSQC spectrum by real-time BIRD homodecoupling

- Figure S11: Effect of NUS in the HD-J-HSQC experiment of strychnine in $\mathrm{PPMA} / \mathrm{CDCl}_{3}$ gel

- Figure S12: Expansion of the HD-J-HSQC spectrum of strychnine in PMMA/CDCl 3 ( Fig. S11B) corresponding to the diastereotopic $\mathrm{CH}_{2}$ protons

- Table S1: Comparison between the experimental ${ }^{1} J_{\mathrm{CH}},{ }^{1} T_{\mathrm{CH}}$ and ${ }^{1} D_{\mathrm{CH}}$ values extracted from a single HD- $J$-HSQC spectrum of strychnine in PMMA/CDCl ${ }_{3}\left({ }^{2} \mathrm{H}\right.$ quadrupolar splitting of $\Delta v_{\mathrm{Q}}=33.4 \mathrm{~Hz}$ ) (this work) with those published previously with a similar sample $\left({ }^{2} \mathrm{H}\right.$ quadrupolar splitting of $\left.\Delta v_{\mathrm{Q}}=36 \mathrm{~Hz}\right)$ using two separate JSB-HSQC measurements... 
- Table S2: RDC-based stereochemical analysis of the 8 possible diastereoisomeric structures of strychnine minimized by Molecular Mechanics and the correct one at a DFT level

- Table S3: Experimental and calculated ${ }^{1} D_{\mathrm{CH}}$ coupling constants of strychnine as a function of the source data and structure calculation (Molecular Mechanics and DFT) 18

- Figure S13: A-B) HD-JSB-HSQC and C-D) HD-J-HSQC spectra of ludartin in isotropic $\mathrm{CDCl}_{3}$ (A and $\mathrm{C}$ ) and anisotropic $\mathrm{PMMA} / \mathrm{CDCl}_{3}$ (B and $\mathrm{D}$ ) sample

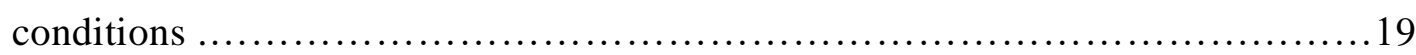

- Table S4. Comparison of RDC values for ludartin extracted from the corresponding HD-JSB-HSQC (Fig. S11B) and HD-J-HSQC (Fig. S11D) spectra 20

- Automated quantitative extraction of ${ }^{1} J_{\mathrm{CH}}$ coupling values from $J$-resolved HSQC spectra

- Pulse program for Bruker AVANCE spectrometers 


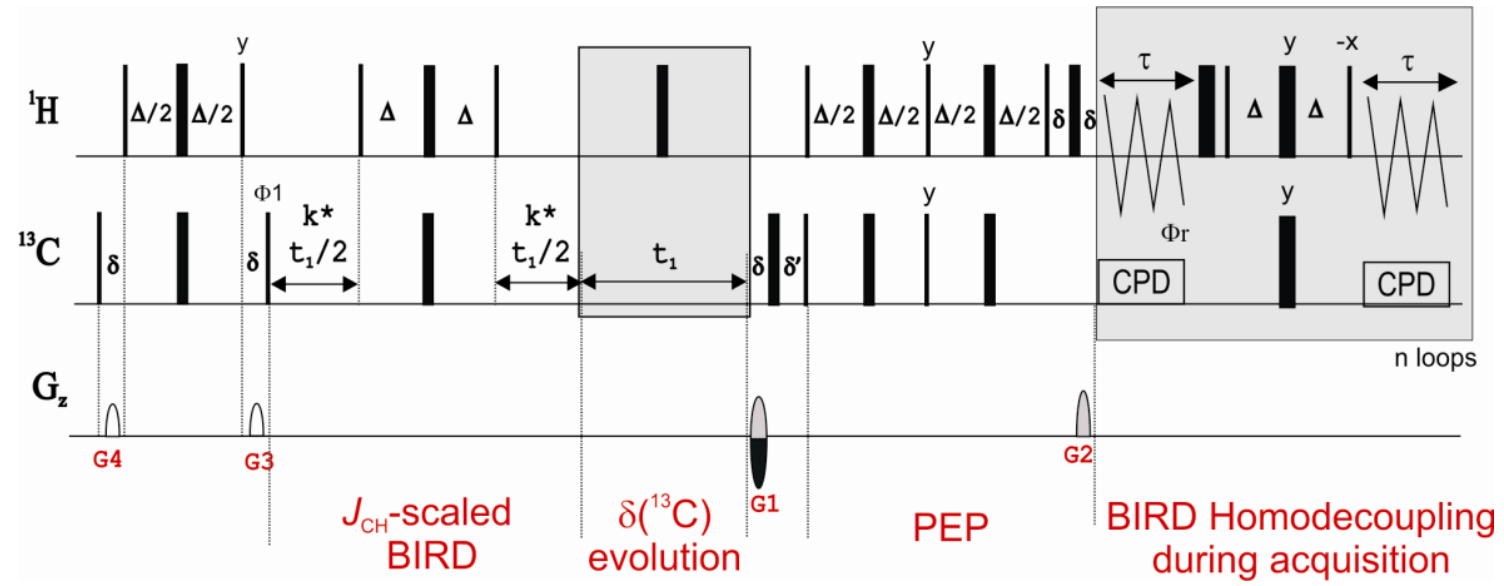

Figure S1: Pulse sequence scheme for real-time BIRD-based homodecoupled $J$-scaled BIRD HSQC (HD-JSB-HSQC) experiment. Narrow and wide filled bars correspond to $90^{\circ}$ and $180^{\circ}$ pulses, respectively, with phase x unless indicated otherwise. All inversion and refocusing $180^{\circ}{ }^{13} \mathrm{C}$ pulses can be applied as adiabatic shaped pulses. The interpulse delay in INEPT and BIRD elements are set to $\Delta=1 /\left(2 * 1 J_{\mathrm{CH}}\right)$. Coherence order selection and echo-antiecho phase sensitive detection in the ${ }^{13} \mathrm{C}$-dimension are achieved with gradient pulses G1 and G2 in the ratio of 80:20.1. Purging gradient pulses G3 and G4 are used with typically of $1 \mathrm{~ms}$ duration followed by a recovery delay of $100 \mu$ s. Each $J$ refocusing block applied during acquisition consists of a BIRD element, a hard $180^{\circ}$ proton pulse, and a data acquisition window. The first and last chunks are half the duration $(\tau=\mathrm{AQ} / 2 \mathrm{n})$ of the remaining chunks $(\mathrm{AQ} / \mathrm{n})$. A basic two-step phase cycle is used: $\phi_{1}=\mathrm{x},-\mathrm{x}$ and $\phi_{\mathrm{rec}}=\mathrm{x},-\mathrm{x}$. 


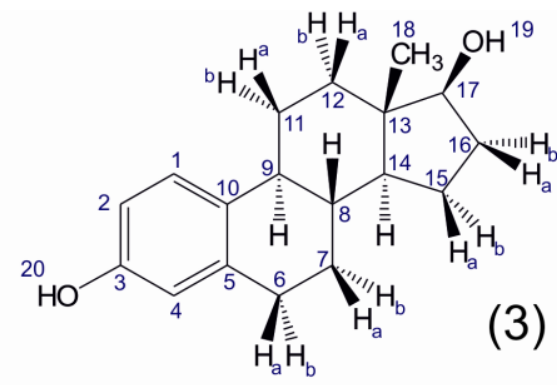

$\begin{array}{ll}\text { A) JSB-HSQC } & \text { B) Homodecoupled }\end{array}$

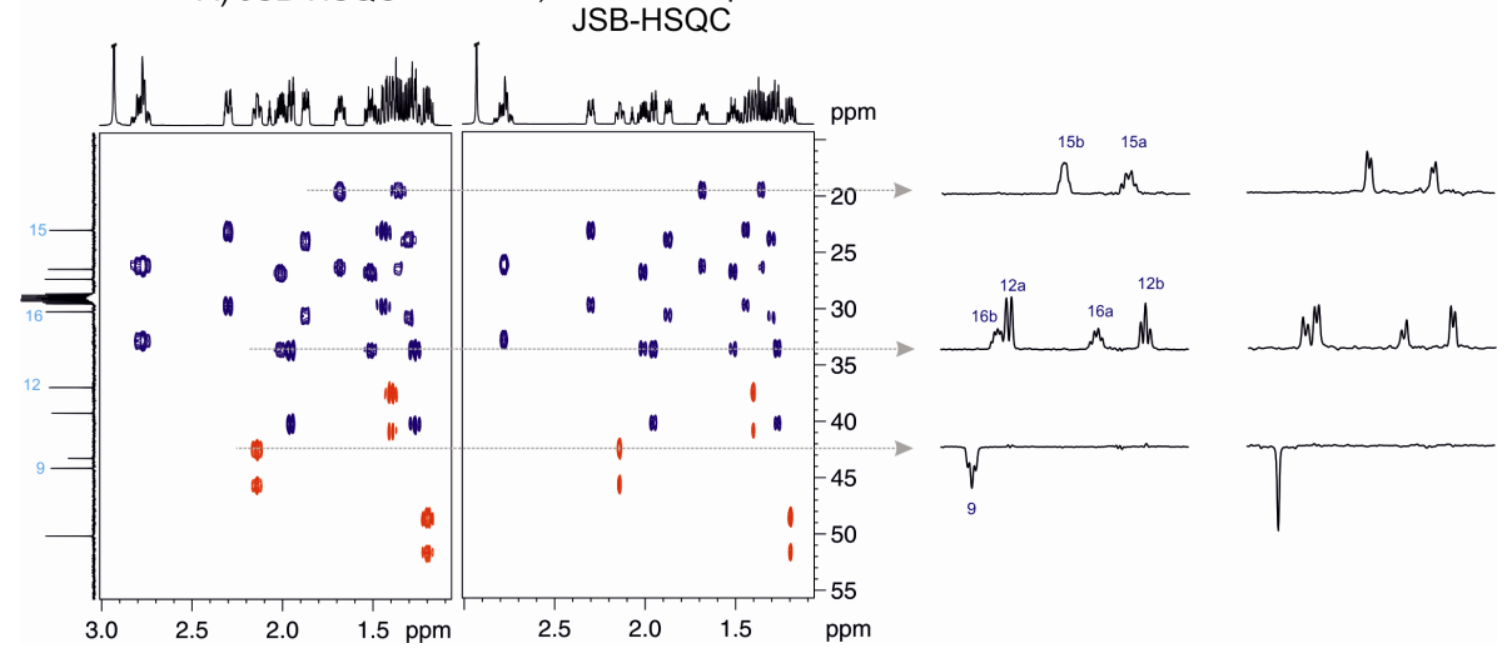

Figure S2: Comparison between the A) conventional JSB-HSQC and B) homodecoupled JSB-HSQC (HD-JSB-HSQC) spectra of an isotropic sample of estradiol (3) acquired with the basic pulse scheme of Fig. S1 (normal FID acquisition vs BIRD-based homodecoupled acquisition, respectively). Right 1D slices from some selected $\mathrm{CH}$ and $\mathrm{CH}_{2}$ correlations are shown to visualize the homodecoupling signal collapsing. Both experiments were acquired and processed under the same experimental conditions: $140 \mathrm{ppm}$ in the ${ }^{13} \mathrm{C}$ dimension, 2 scans per $t_{1}$ increment, $128 t_{1}$ increments and a $J$-scaling factor of 4 . The experimental time for each $2 \mathrm{D}$ spectrum was 5 min $32 \mathrm{~s}$ for A) and 6 min $12 \mathrm{~s}$ for B). 

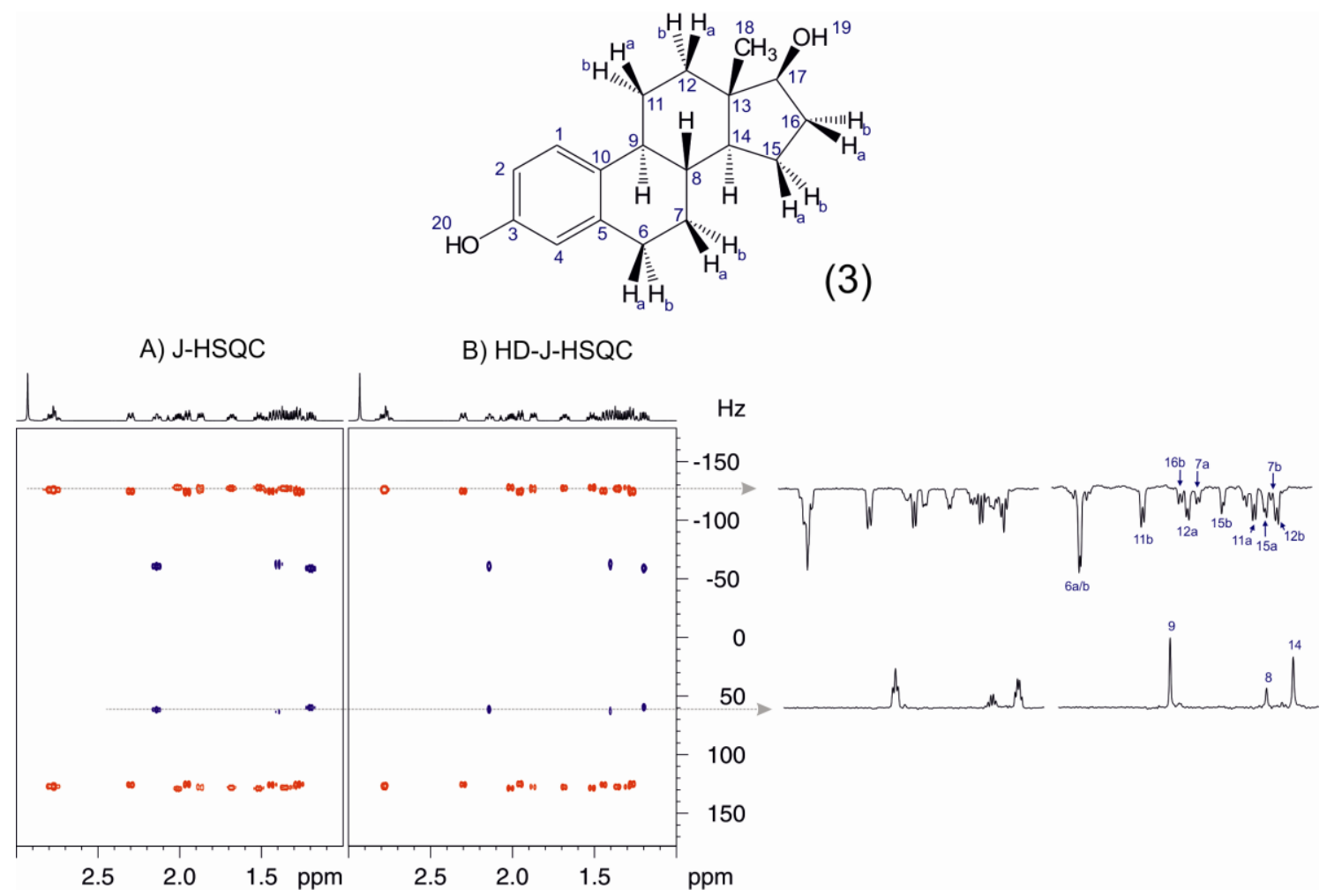

Figure S3: Comparison between the A) conventional $J$-HSQC and B) HD-J-HSQC spectra of an isotropic sample of estradiol (3) acquired with the basic pulse scheme of Fig. 1 (normal FID acquisition vs BIRD-based homodecoupled acquisition, respectively). Right $1 \mathrm{D}$ slices from some selected $\mathrm{CH}$ and $\mathrm{CH}_{2}$ correlations are shown to visualize the homodecoupling signal collapsing. Both experiments were acquired and processed under the same experimental conditions: $4 \mathrm{ppm}$ in the ${ }^{13} \mathrm{C}$ dimension, 2 scans per $t_{1}$ increment, and $128 t_{1}$ increments. The experimental time for each $2 \mathrm{D}$ spectrum was $5 \operatorname{min~} 46 \mathrm{~s}$ for $\mathrm{A}$ ) and $6 \mathrm{~min} 26 \mathrm{~s}$ for $\mathrm{B}$ ). These spectra are equivalent to those of Fig. S2. 


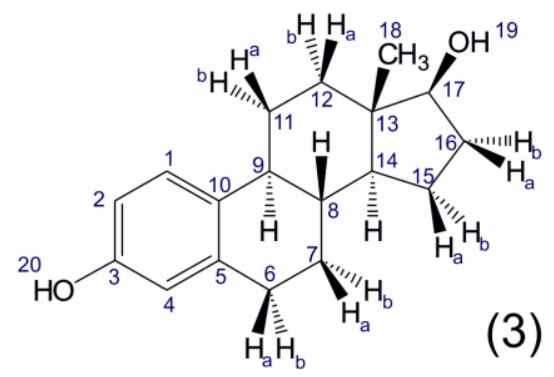
A) $k=1$
B) $k=2$
C) $k=4$
D) $k=8$

E)
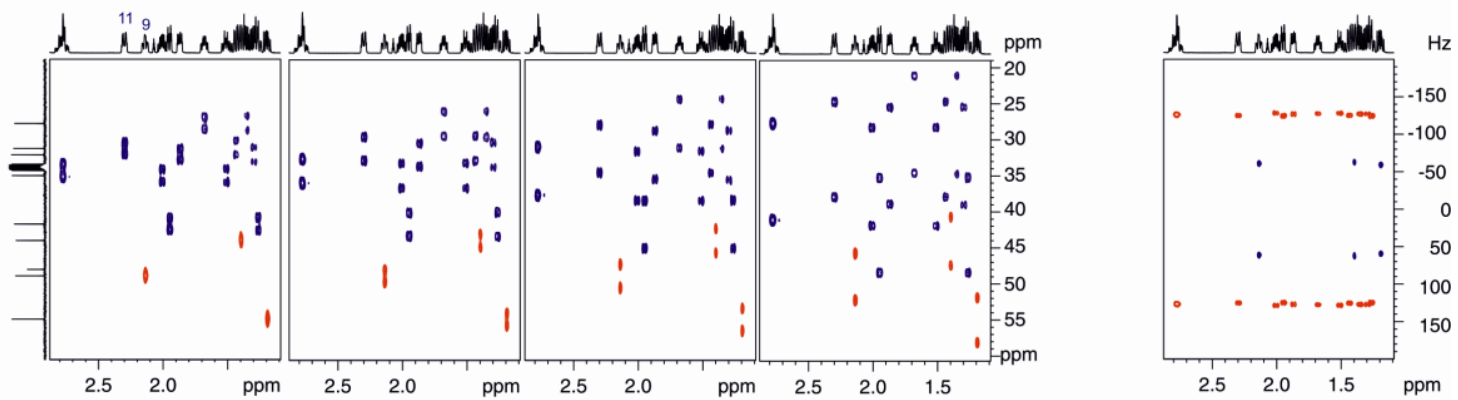

FID res: $\quad 19.5 \mathrm{~Hz}$

$19.5 \mathrm{~Hz}$

$19.5 \mathrm{~Hz}$

$19.5 \mathrm{~Hz}$

$0.5 \mathrm{~Hz}$

${ }^{1} \mathrm{~J}_{\mathrm{CH}}=244.3 \mathrm{~Hz}$

${ }^{1} \mathrm{~J}_{\mathrm{CH}}=251.4 \mathrm{~Hz}$

${ }^{1} \mathrm{~J}_{\mathrm{CH}}=250.2 \mathrm{~Hz}$

${ }^{1} \mathrm{~J}_{\mathrm{CH}}=249.3 \mathrm{~Hz}$
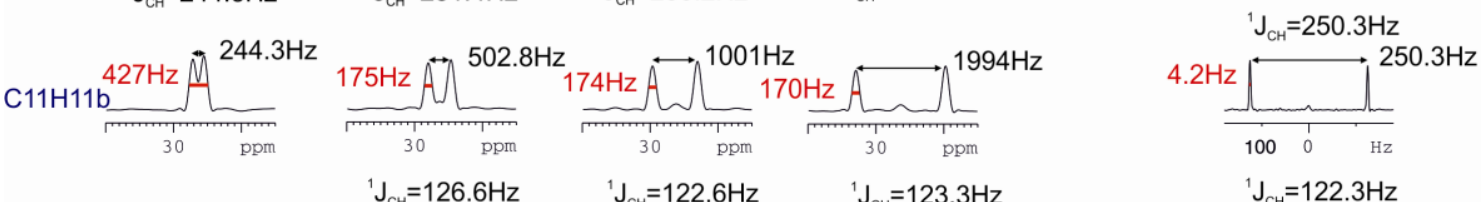

C9H9 $207 \mathrm{~Hz}$

$$
{ }^{1} J_{\mathrm{CH}}=126.6 \mathrm{~Hz}
$$

${ }^{1} \mathrm{~J}_{\mathrm{CH}}=122.6 \mathrm{~Hz}$

${ }^{1} \mathrm{~J}_{\mathrm{CH}}=123.3 \mathrm{~Hz}$

${ }^{1} \mathrm{~J}_{\mathrm{CH}}=122.3 \mathrm{~Hz}$

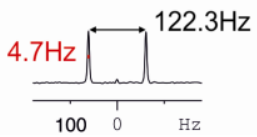

Figure S4: Comparison between several A-D) HD-JSB-HSQC ( $J$-scaling factors of $\mathrm{k}=$ 1,2,4 and 8 respectively) and E) the analog HD-J-HSQC spectra of an isotropic sample of estradiol (3) . All spectra have been acquired under the same experimental conditions (128 $t_{1}$ increments) and with similar experimental times. Below are the corresponding $\mathrm{F}_{1}$ projections at the specific $\mathrm{C} 11 \mathrm{H} 11 \mathrm{~b}$ and $\mathrm{C} 9 \mathrm{H} 9$ correlations to compare experimental splittings, spectral resolution and linewidths. Note that improved resolution and linewidths resolution by a factor of 40 is achieved in the HD-J-HSQC spectrum, rendering a much more accurate determination of ${ }^{1} J_{\mathrm{CH}}$. 

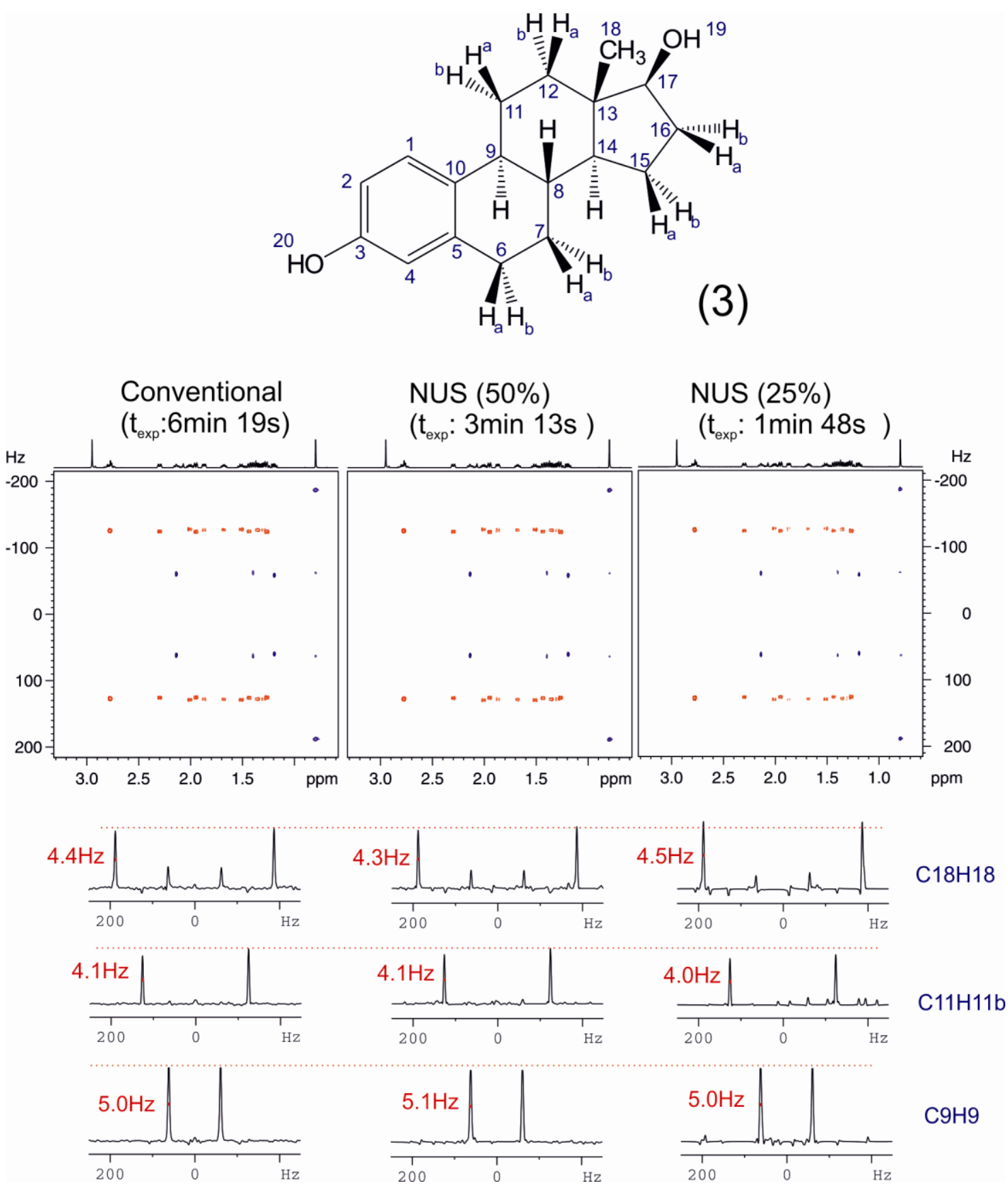

Figure S5: Effect of nonuniform sampling (NUS) on the experimental time of the HD$J$-HSQC experiment. Comparison between several HD-J-HSQC spectra of an isotropic sample of estradiol (3) acquired with (left) conventional uniform sampling (128 $t_{1}$ increments) and with (center) $50 \%$ and (right) $25 \%$ of NUS. Below are some $\mathrm{F}_{1}$ columns extracted at specific proton resonances. It shows that similar spectral quality, signal resolution and linewidths are achieved in all spectra, allowing an important spectrometer time saving factor of 4 when using $25 \%$ of NUS. Also note that signal sensitivity is retained in all equivalent spectra. 


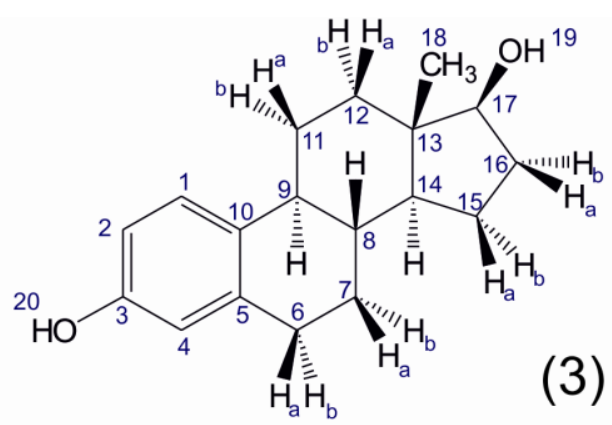

Conventional $(\mathrm{TD}=128) \quad \mathrm{NUS}(50 \%)(\mathrm{TD}=256) \quad \mathrm{NUS}(25 \%)(\mathrm{TD}=512)$
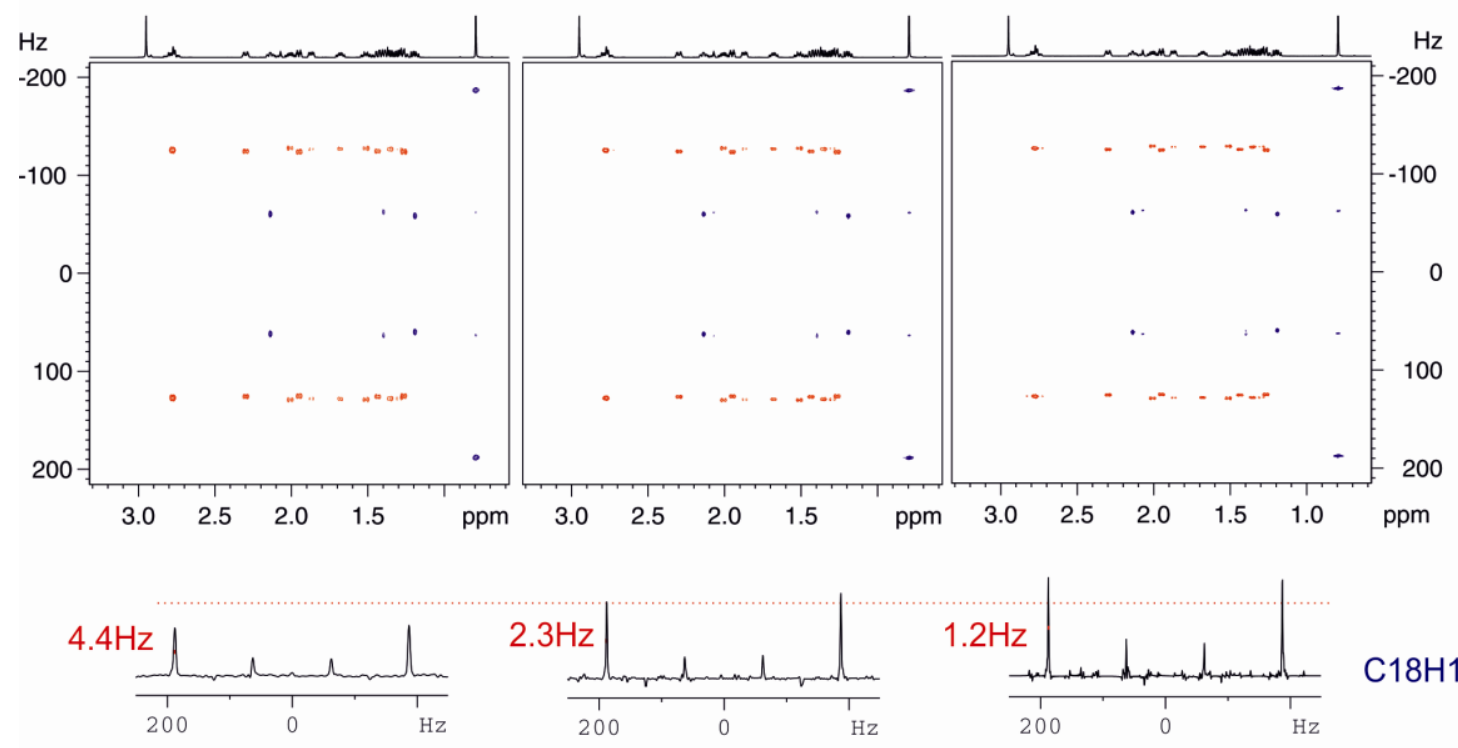

$\mathrm{C} 18 \mathrm{H} 18$
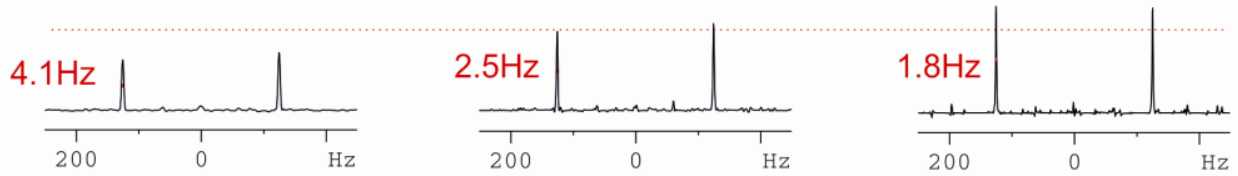

$\mathrm{C} 11 \mathrm{H} 11 \mathrm{~b}$
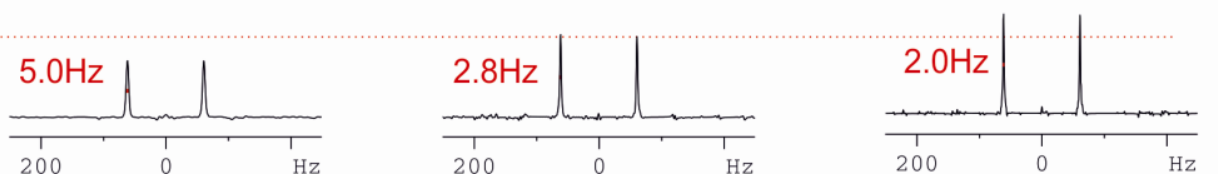

$\mathrm{C9H9}$

Figure S6: Effect of NUS on the signal resolution of HD-J-HSQC experiment. Comparison between several HD-J-HSQC spectra of an isotropic sample of estradiol (3) acquired with (left) conventional uniform sampling (128 $t_{1}$ increments) and with (center) $50 \%$ (of $256 t_{1}$ increments) and (right) $25 \%$ (of $512 t_{1}$ increments) of NUS. All experiments were collected in the same experimental time of 6 min $3 \mathrm{~s}$. Below are some $\mathrm{F}_{1}$ columns extracted at specific proton resonances. It is shown that improved signal resolution, sharper linewidths and better signal sensitivity are achieved with increased use of NUS. 

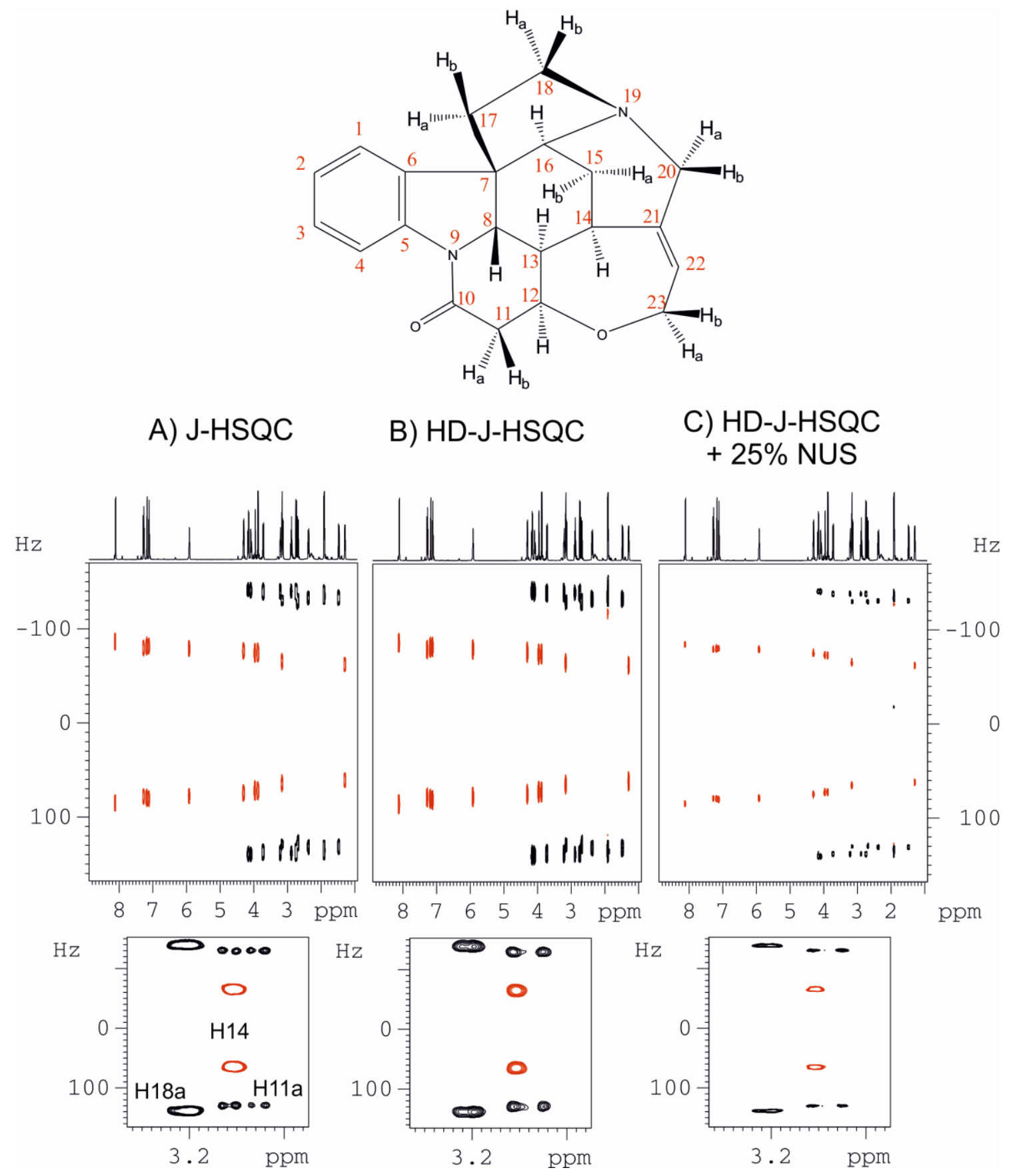

Figure S7: A) Conventional $J$-HSQC, B) equivalent HD- $J$-HSQC (with real-time homodecoupling during acquisition) and C) HD-J-HSQC spectra using 25\% NUS of an isotropic sample of strychnine (1) in $\mathrm{CDCl}_{3}$. All spectra have been acquired under the same conditions and experimental time. 


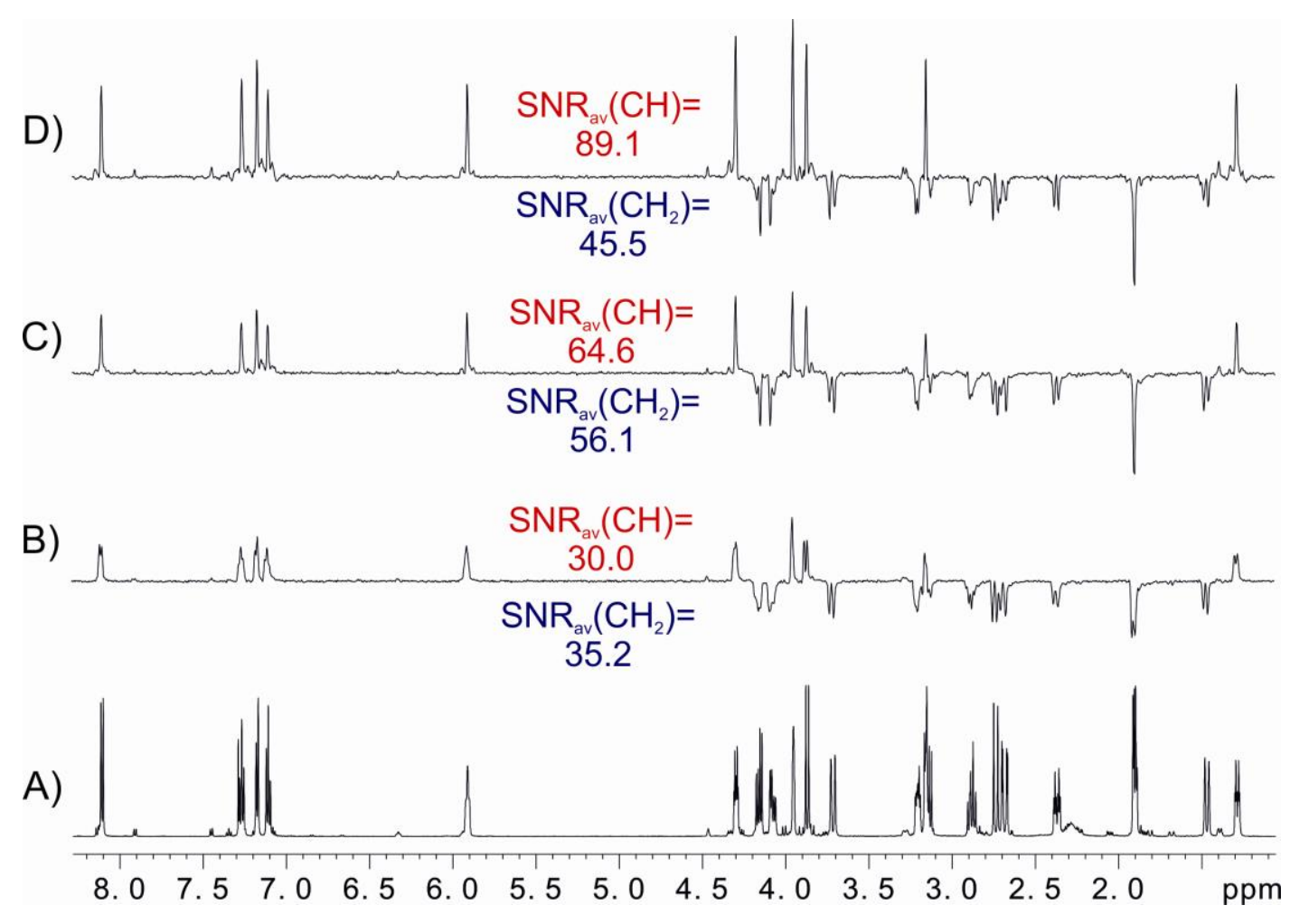

Figure S8: Edited $\mathrm{CH} / \mathrm{CH}_{2}$ internal $\mathrm{F}_{1}$ projections extracted from B) $J$-HSQC with INEPT, C) HD- $J$-HSQC with INEPT and D) HD-J-HSQC with PEP transfer spectra of strychnine in $\mathrm{CDCl}_{3}$. The corresponding averaged $\mathrm{SNR}$ values for each edited $\mathrm{CH}$ (red) and $\mathrm{CH}_{2}$ (blue) subgroups of protons are shown in B-D). 


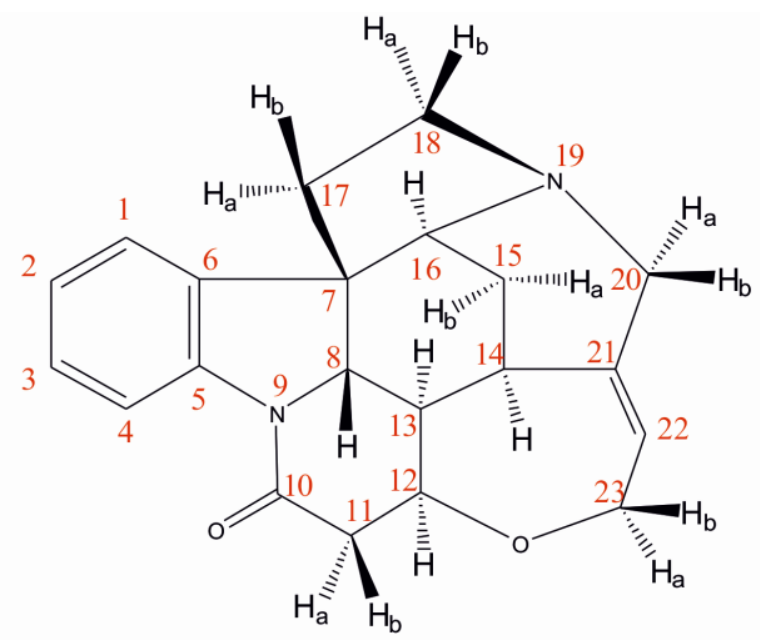

A) J-HSQC

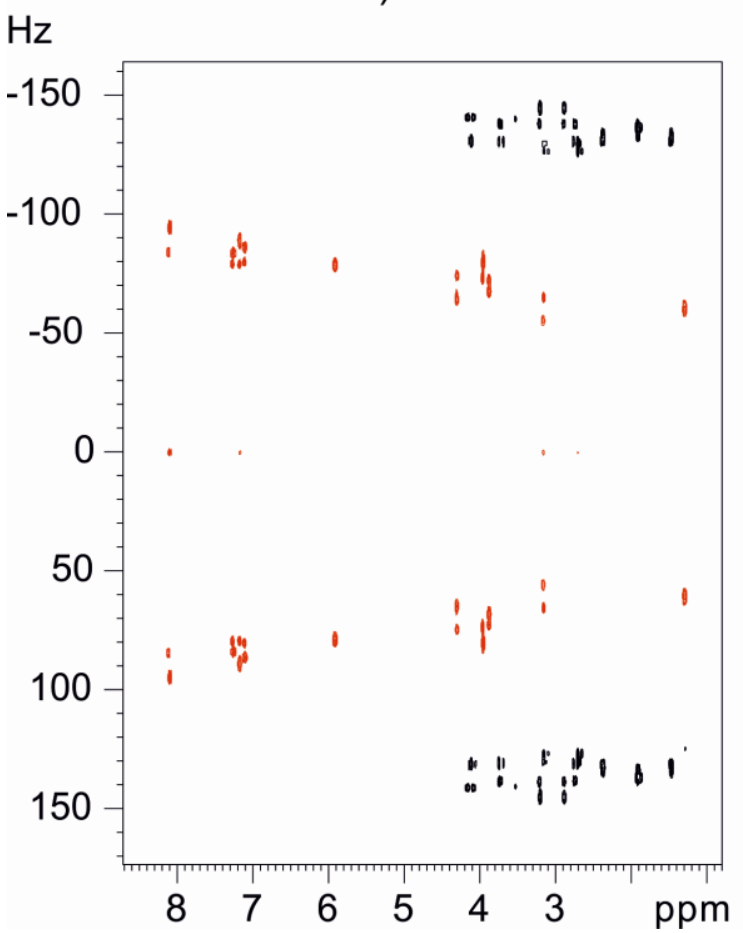

B) HD-J-HSQC

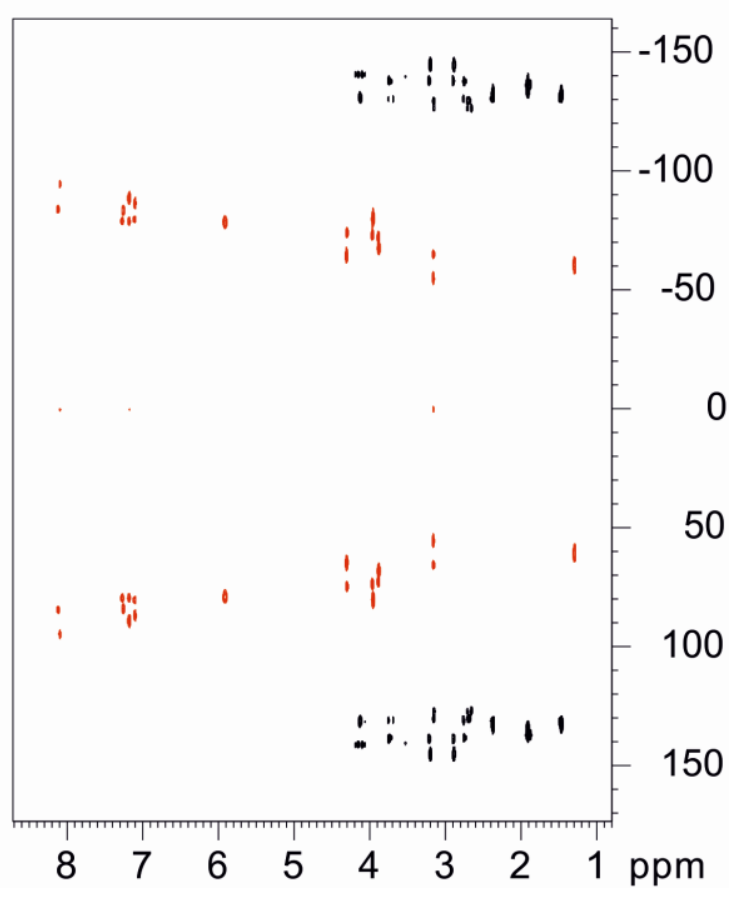

Figure S9: (A) 2D $J$-HSQC and (B) 2D HD-J-HSQC spectra of an anisotropic sample of strychnine (1) dissolved in PMMA/CDCl 3 . Experimental details: spectral width $\left(\mathrm{F}_{1}\right)$ $=4 \mathrm{ppm}$, number of complex data points in $\mathrm{F}_{2}=2048$, number of $t_{1}$ increments $=512$, FID resolution $=2.35 \mathrm{~Hz}$, number of scans $=4$, relaxation delay $=1 \mathrm{~s}$, experimental time $=53 \min 13 \mathrm{~s}$, spectral resolution after zero filling to $2 \mathrm{k}$ in $\mathrm{F}_{2}=0.29 \mathrm{~Hz}$. Data acquisition was divided into 8 chunks with $\tau=10.3 \mathrm{~ms}$ each one. 


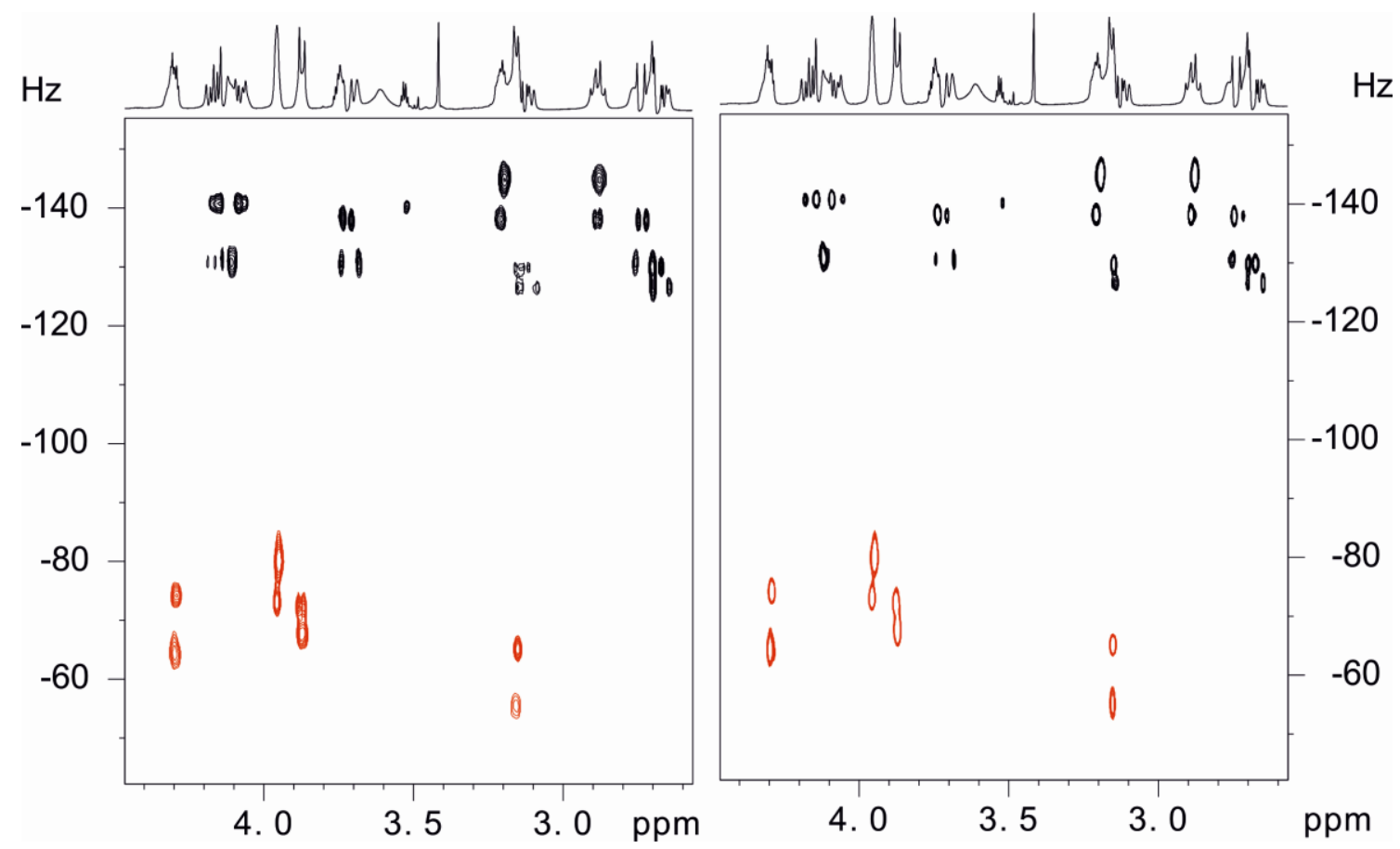

Figure S10: Expansions extracted from spectra of Fig. S9 that show the multiplet $J$ pattern simplification achieved in the HD-J-HSQC spectrum by real-time BIRD homodecoupling. All $\mathrm{CH}$ cross-peaks (red) are converted to singlet resonances whereas diastereotopic $\mathrm{CH}_{2}$ protons (black) are simplified to doublets. 

A) HD-J-HSQC
$53 \min 13 \mathrm{~s}$
B) HD-J-HSQC $25 \%$ NUS
$11 \mathrm{~min} 34 \mathrm{~s}$
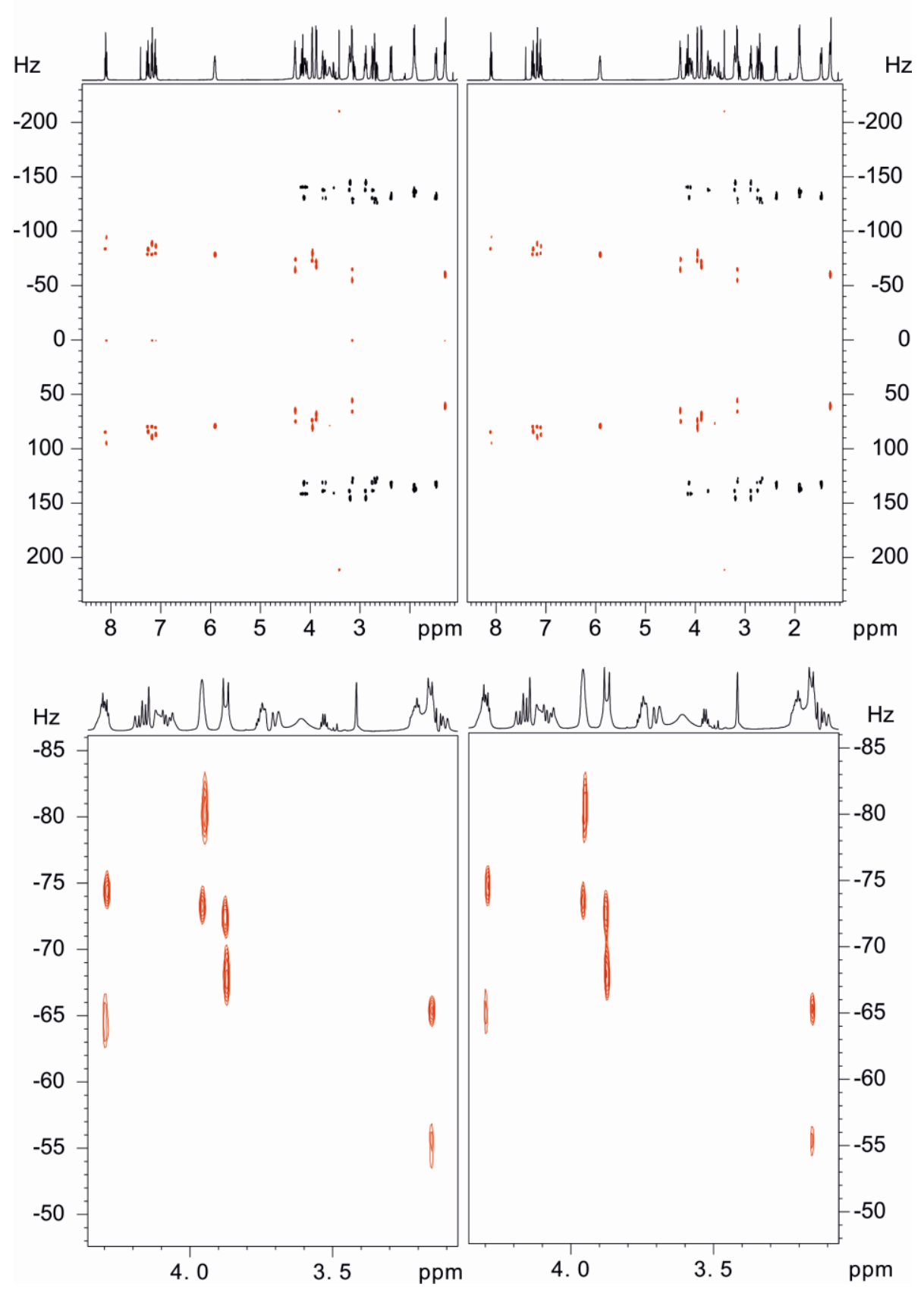

Figure S11: Effect of NUS in the HD- $J$-HSQC experiment of strychnine (1) in PMMA/CDCl 3 gel. Both experiments were recorded under the same experimental conditions: A) Conventional sampling in $t_{1}\left(512 t_{1}\right.$ increments, experimental time $=53$ min $13 \mathrm{~s}$ and B) with $25 \%$ of NUS (512 $t_{1}$ increments, experimental time 11 min $34 \mathrm{~s}$ ). Note that very accurate ${ }^{1} D_{\mathrm{CH}}$ values can be measured directly from each $\mathrm{CH}$ resonance. Expanded region corresponding to the upfield components of the $\mathrm{CH}$ protons are shown in the spectra below. 


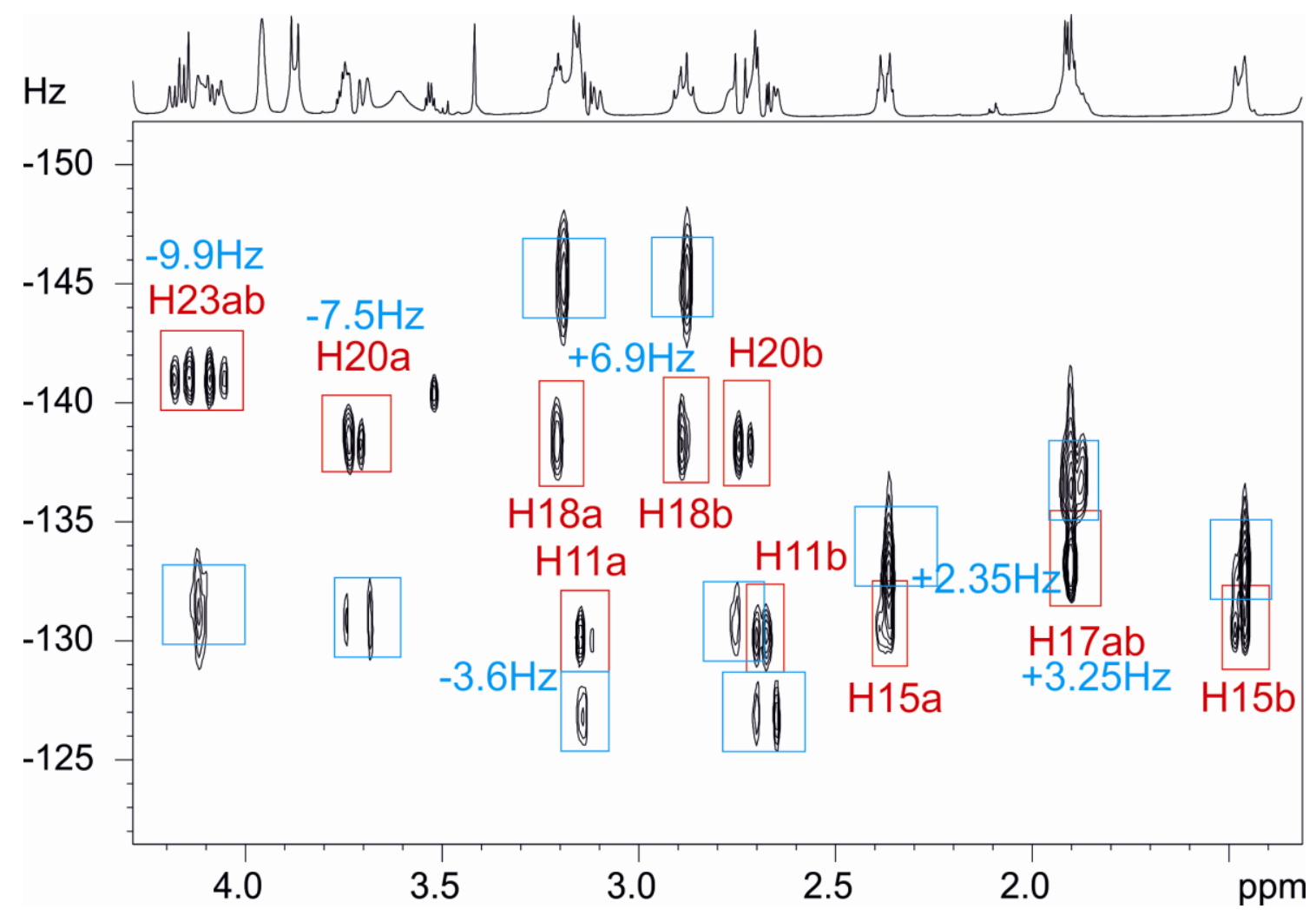

Figure S12: Expanded region corresponding to the upfield components of the diastereotopic $\mathrm{CH}_{2}$ protons in the HD-J-HSQC spectrum of strychnine in $\mathrm{PMMA} \mathrm{CDCl}_{3}$ (see Fig. S11B). Red and blue boxes represent the isotropic (provides ${ }^{1} J_{\mathrm{CH}}$ ) and anisotropic (provides ${ }^{1} T_{\mathrm{CH}}$ ) components for each individual proton resonance. Note that very accurate signs and magnitudes of $\left({ }^{1} D_{\mathrm{Cha}}+{ }^{1} D_{\mathrm{CHb}}\right) / 2$ values can be measured directly from each pair of iso/aniso components. 
Table S1: Comparison between the experimental ${ }^{1} J_{\mathrm{CH}},{ }^{1} T_{\mathrm{CH}}$ and ${ }^{1} D_{\mathrm{CH}}$ values extracted from a single HD- $J$-HSQC spectrum of strychnine in $\mathrm{PMMA} / \mathrm{CDCl}_{3}\left({ }^{2} \mathrm{H}\right.$ quadrupolar splitting of $\Delta v_{\mathrm{Q}}=33.4 \mathrm{~Hz}$ ) (this work) with those published previously with a similar sample $\left({ }^{2} \mathrm{H} \text { quadrupolar splitting of } \Delta v_{\mathrm{Q}}=36 \mathrm{~Hz}\right)^{\mathrm{b}}$ using two separate JSB-HSQC measurements.

\begin{tabular}{|c|c|c|c|c|c|}
\hline \multirow[b]{2}{*}{$\mathrm{CH}$ pairs } & \multicolumn{2}{|c|}{ JSB-HSQC $^{\mathrm{b}}$} & \multicolumn{3}{|c|}{ HD- $J$-HSQC } \\
\hline & $\begin{array}{c}\text { Isotropic } \\
{ }^{1} J_{\mathrm{CH}} \\
\mathrm{Hz} \\
\end{array}$ & $\begin{array}{c}\text { Anisotropic } \\
{ }^{1} D_{\mathrm{CH}} \\
\mathrm{Hz}\end{array}$ & $\begin{array}{l}\text { Aniso } \\
{ }^{1} J_{\mathrm{CH}} \\
\mathrm{Hz}\end{array}$ & $\begin{array}{l}\text { c (this wo } \\
{ }^{1} T_{\mathrm{CH}} \\
\mathrm{Hz}\end{array}$ & $\begin{array}{l}{ }^{1} D_{\mathrm{CH}} \\
\mathrm{Hz}\end{array}$ \\
\hline $\mathrm{C} 1 \mathrm{H} 1$ & 158.38 & 20.97 & 158.6 & 178.2 & 19.6 \\
\hline $\mathrm{C} 2 \mathrm{H} 2$ & 161.70 & 12.29 & 160 & 172.3 & 12.3 \\
\hline $\mathrm{C} 3 \mathrm{H} 3$ & 160.19 & 10.04 & 158.9 & 167.5 & 8.6 \\
\hline $\mathrm{C} 4 \mathrm{H} 4$ & 168.35 & 21.58 & 168.6 & 189.2 & 20.6 \\
\hline $\mathrm{C} 8 \mathrm{H} 8$ & 144.91 & -9.75 & 144.6 & 135.9 & -8.7 \\
\hline $\mathrm{C} 11 \mathrm{H} 11 \mathrm{a}+\mathrm{C} 11 \mathrm{H} 11 \mathrm{~b}$ & 260.84 & $-3.46^{\mathrm{a}}$ & 260.4 & 253.2 & $-3.6^{\mathrm{a}}$ \\
\hline $\mathrm{C} 12 \mathrm{H} 12$ & 149.03 & -20.59 & 149.3 & 128.8 & -20.5 \\
\hline $\mathrm{C} 13 \mathrm{H} 13$ & 124.88 & -4.87 & 124.7 & 119.6 & -5.1 \\
\hline $\mathrm{C} 14 \mathrm{H} 14$ & 131.03 & -20.66 & 131.1 & 111.8 & -19.3 \\
\hline $\mathrm{C} 15 \mathrm{H} 15 \mathrm{a}+\mathrm{C} 15 \mathrm{H} 15 \mathrm{~b}$ & 260.66 & $2.54^{\mathrm{a}}$ & 260.82 & 265.5 & $2.34^{\mathrm{a}}$ \\
\hline C16H6 & 146.55 & 14.04 & 146.9 & 160.5 & 13.6 \\
\hline $\mathrm{C} 17 \mathrm{H} 17 \mathrm{a}+\mathrm{C} 17 \mathrm{H} 17 \mathrm{~b}$ & 265.92 & $1.38^{\mathrm{a}}$ & 266.5 & 273 & $3.25^{\mathrm{a}}$ \\
\hline $\mathrm{C} 18 \mathrm{H} 18 \mathrm{a}+\mathrm{C} 18 \mathrm{H} 18 \mathrm{~b}$ & 276.20 & $6,89^{a}$ & 276.4 & 290.2 & $6.9^{\mathrm{a}}$ \\
\hline $\mathrm{C} 20 \mathrm{H} 20 \mathrm{a}+\mathrm{C} 20 \mathrm{H} 20 \mathrm{~b}$ & 276.56 & $-7.39^{\mathrm{a}}$ & 276.7 & 261.7 & $-7.5^{\mathrm{a}}$ \\
\hline $\mathrm{C} 22 \mathrm{H} 22$ & 158.91 & -1.06 & & c & \\
\hline $\mathrm{C} 23 \mathrm{H} 23 \mathrm{a}+\mathrm{C} 23 \mathrm{H} 23 \mathrm{~b}$ & 282.42 & $-10.3^{a}$ & 282.1 & 262.3 & $-9.9^{a}$ \\
\hline
\end{tabular}

${ }^{\text {a }}$ These values correspond to $\left({ }^{1} D_{\mathrm{Cha}}+{ }^{1} D_{\mathrm{CHb}}\right) / 2$ to be used in MSpin.

${ }^{\mathrm{b}}$ Experimental data obtained from J. D. Snider et al. Magn. Reson. Chem. 50 (2012) S86.

${ }^{\mathrm{c}}$ Not measured 
Table S2: RDC-based stereochemical analysis of the 8 possible diastereoisomeric structures of strychnine minimized by Molecular Mechanics (MMFFs) and the correct one at a DFT level:

\begin{tabular}{|c|c|c|c|c|c|c|}
\hline & \multirow[b]{2}{*}{ Isomer } & \multicolumn{5}{|c|}{ Q factors } \\
\hline & & $\begin{array}{l}\text { From } \\
\text { ref. } 1^{\mathrm{a}, \mathrm{c}}\end{array}$ & $\begin{array}{l}\text { This work } \\
\text { from data } \\
\text { of ref. } 1^{\mathrm{a}, \mathrm{c}}\end{array}$ & $\begin{array}{c}\text { This work } \\
\text { from HD- } \\
J \text {-HSQC } \\
\text { data }^{\mathrm{b}, \mathrm{c}}\end{array}$ & $\begin{array}{l}\text { This work } \\
\text { from data } \\
\text { of ref. } 1^{\mathrm{a}, \mathrm{d}}\end{array}$ & $\begin{array}{c}\text { This work } \\
\text { from HD- } \\
J \text {-HSQC } \\
\text { data }^{\text {b,d }}\end{array}$ \\
\hline 1 & $7 R, 8 R, 12 R, 13 R, 14 R, 16 S$ & 0.294 & 0.313 & 0.313 & & \\
\hline 2 & $7 R, 8 R, 12 S, 13 R, 14 R, 16 S$ & 0.370 & 0.396 & 0.407 & & \\
\hline 3 & $7 R, 8 R, 12 R, 13 S, 14 R, 16 S$ & 0.347 & 0.347 & 0.294 & & \\
\hline 4 & $7 R, 8 R, 12 S, 13 S, 14 R, 16 S$ & 0.320 & 0.320 & 0.268 & & \\
\hline 5 & $7 R, 8 S, 12 R, 13 R, 14 R, 16 S$ & 0.115 & 0.122 & 0.118 & & \\
\hline 6 & $7 R, 8 S, 12 S, 13 R, 14 R, 16 S$ & 0.045 & 0.054 & 0.061 & 0.045 & 0.031 \\
\hline 7 & $7 R, 8 S, 12 R, 13 S, 14 R, 16 S$ & 0.370 & 0.371 & 0.332 & & \\
\hline 8 & $7 R, 8 S, 12 S, 13 S, 14 R, 16 S$ & 0.242 & 0.247 & 0.219 & & \\
\hline
\end{tabular}

${ }^{a}$ Experimental data obtained from [1] J. D. Snider et al. Magn. Reson. Chem. 50 (2012) $\mathrm{S} 86\left(\Delta v_{\mathrm{Q}}=36 \mathrm{~Hz}\right)$

${ }^{\mathrm{b}}$ Experimental data obtained from HD-J-HSQC data $\left(\Delta v_{\mathrm{Q}}=33.6 \mathrm{~Hz}\right)$

${ }^{c}$ Structures calculated by Molecular Mechanics (MMFFs)

${ }^{\mathrm{d}}$ Structure calculated at a DFT level (B3LYP/6-31G*) 
Table S3: Experimental and calculated ${ }^{1} D_{\mathrm{CH}}$ coupling constants of strychnine as a function of the source data and structure calculation (Molecular Mechanics and DFT)

\begin{tabular}{|c|c|c|c|c|c|c|}
\hline & \multicolumn{3}{|c|}{ This work from data of ref. $1^{a}$} & \multicolumn{3}{|c|}{ This work from HD-J-HSQC data ${ }^{b}$} \\
\hline & $\begin{array}{c}\operatorname{Exp}^{1} D_{\mathrm{CH}} \\
(\mathrm{Hz})^{\mathrm{a}}\end{array}$ & $\begin{array}{c}\text { Calc }{ }^{1} D_{\mathrm{CH}} \\
(\mathrm{Hz})^{\mathrm{c}}\end{array}$ & $\begin{array}{c}\text { Calc }{ }^{1} D_{\mathrm{CH}} \\
(\mathrm{Hz}){ }^{\mathrm{d}}\end{array}$ & $\begin{array}{c}\operatorname{Exp}{ }^{1} D_{\mathrm{CH}} \\
(\mathrm{Hz})^{\mathrm{b}}\end{array}$ & $\begin{array}{c}\text { Calc }{ }^{1} D_{\mathrm{CH}} \\
(\mathrm{Hz})^{\mathrm{c}}\end{array}$ & $\begin{array}{c}\text { Calc }{ }^{1} D_{\mathrm{CH}} \\
(\mathrm{Hz}){ }^{\mathrm{d}}\end{array}$ \\
\hline $\mathrm{C} 1 \mathrm{H} 1$ & 20.97 & 21.11 & 20.97 & 19.60 & 19.98 & 19.91 \\
\hline $\mathrm{C} 2 \mathrm{H} 2$ & 12.29 & 12.18 & 12.53 & 12.30 & 12.08 & 12.45 \\
\hline $\mathrm{C} 3 \mathrm{H} 3$ & 10.04 & 9.71 & 9.68 & 8.60 & 8.48 & 8.64 \\
\hline $\mathrm{C} 4 \mathrm{H} 4$ & 21.58 & 21.08 & 21.27 & 20.60 & 19.93 & 20.20 \\
\hline C8H8 & -9.75 & -10.04 & -10.08 & -8.70 & -9.06 & -9.11 \\
\hline $\mathrm{C} 11 \mathrm{H} 11 \mathrm{a}$ & -3.46 & -5.11 & -4.01 & -3.60 & -4.90 & -3.73 \\
\hline $\mathrm{C} 11 \mathrm{H} 11 \mathrm{~b}$ & -3.46 & -5.11 & -4.01 & -3.60 & -4.90 & -3.73 \\
\hline $\mathrm{C} 12 \mathrm{H} 12$ & -20.59 & -19.64 & -20.63 & -20.50 & -19.04 & -19.85 \\
\hline $\mathrm{C} 13 \mathrm{H} 13$ & -4.87 & -4.95 & -5.23 & -5.10 & -5.16 & -5.04 \\
\hline C14H14 & -20.66 & -21.76 & -20.68 & -19.30 & -20.85 & -19.89 \\
\hline $\mathrm{C} 15 \mathrm{H} 15 \mathrm{a}$ & 2.54 & 2.45 & 2.76 & 2.34 & 2.21 & 2.47 \\
\hline $\mathrm{C} 15 \mathrm{H} 15 \mathrm{~b}$ & 2.54 & 2.45 & 2.76 & 2.34 & 2.21 & 2.47 \\
\hline $\mathrm{C} 16 \mathrm{H} 6$ & 14.04 & 13.93 & 13.76 & 13.60 & 13.93 & 13.69 \\
\hline $\mathrm{C} 17 \mathrm{H} 17 \mathrm{a}$ & 1.38 & 2.64 & 3.11 & 3.25 & 3.14 & 3.35 \\
\hline $\mathrm{C} 17 \mathrm{H} 17 \mathrm{~b}$ & 1.38 & 2.64 & 3.11 & 3.25 & 3.14 & 3.35 \\
\hline $\mathrm{C} 18 \mathrm{H} 18 \mathrm{a}$ & 6.89 & 7.04 & 7.03 & 6.90 & 6.60 & 6.79 \\
\hline $\mathrm{C} 18 \mathrm{H} 18 \mathrm{~b}$ & 6.89 & 7.04 & 7.03 & 6.90 & 6.60 & 6.79 \\
\hline $\mathrm{C} 20 \mathrm{H} 20 \mathrm{a}$ & -7.39 & -6.86 & -7.05 & -7.50 & -6.28 & -6.54 \\
\hline $\mathrm{C} 20 \mathrm{H} 20 \mathrm{~b}$ & -7.39 & -6.86 & -7.05 & -7.50 & -6.28 & -6.54 \\
\hline $\mathrm{C} 22 \mathrm{H} 22$ & -1.06 & -0.58 & -0.05 & $* * *$ & -1.35 & -0.40 \\
\hline $\mathrm{C} 23 \mathrm{H} 23 \mathrm{a}$ & -10.30 & -10.40 & -10.49 & -9.90 & -9.67 & -9.71 \\
\hline $\mathrm{C} 23 \mathrm{H} 23 \mathrm{~b}$ & -10.30 & -10.40 & -10.49 & -9.90 & -9.67 & -9.71 \\
\hline
\end{tabular}

${ }^{a}$ Experimental data obtained from [1] J. D. Snider et al. Magn. Reson. Chem. 50 (2012)

$\mathrm{S} 86\left(\Delta v_{\mathrm{Q}}=36 \mathrm{~Hz}\right)$

${ }^{\mathrm{b}}$ Experimental data obtained from HD-J-HSQC data $\left(\Delta v_{\mathrm{Q}}=33.6 \mathrm{~Hz}\right)$

${ }^{c}$ Structures calculated by Molecular Mechanics

${ }^{\mathrm{d}}$ Structure calculated at a DFT (B3LYP/6-31G*) level 


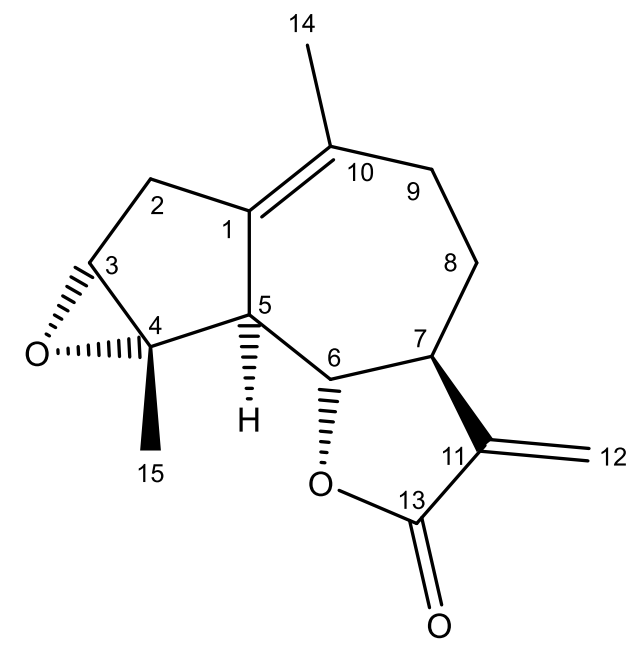

A)

B)
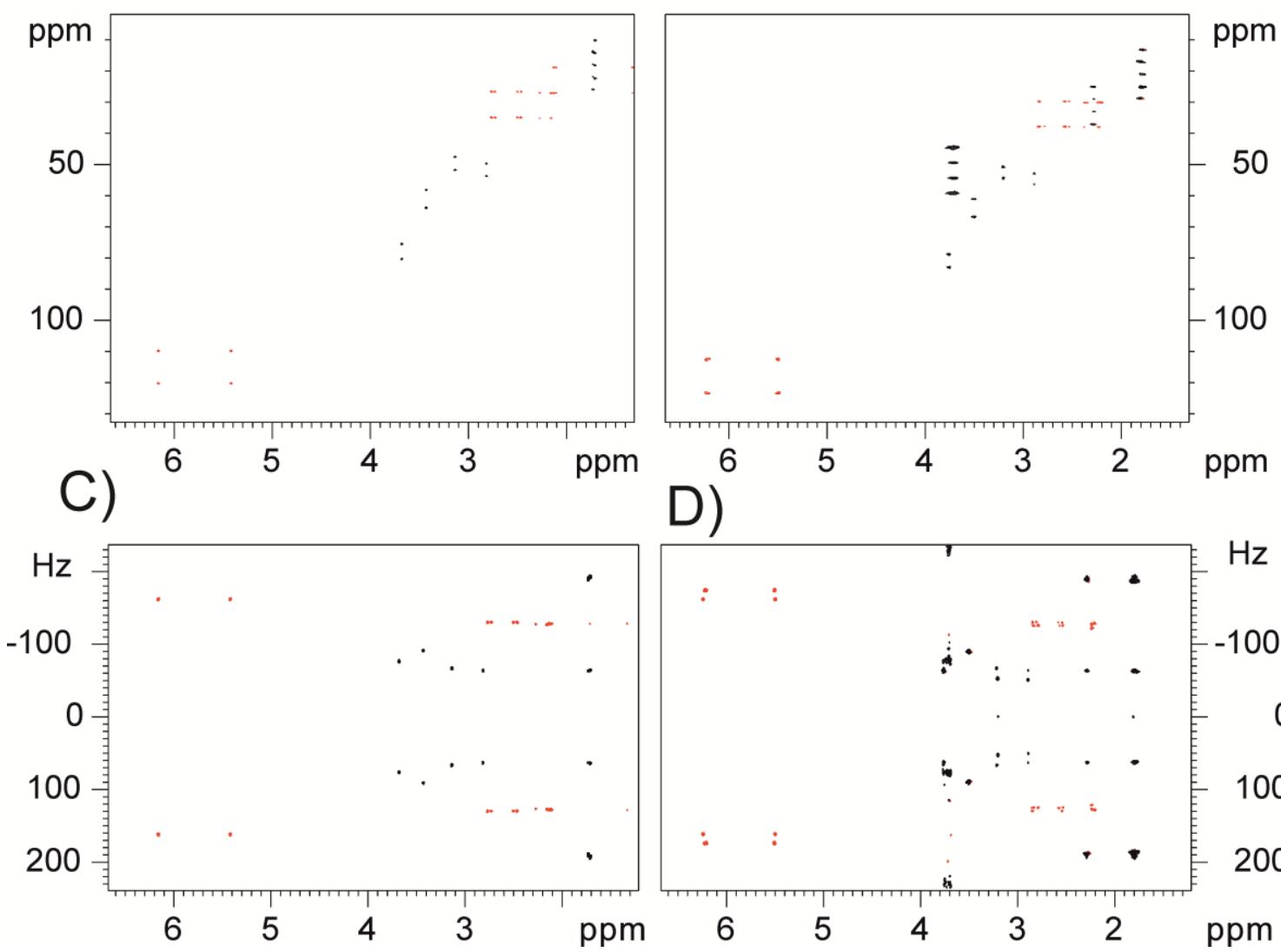

D)

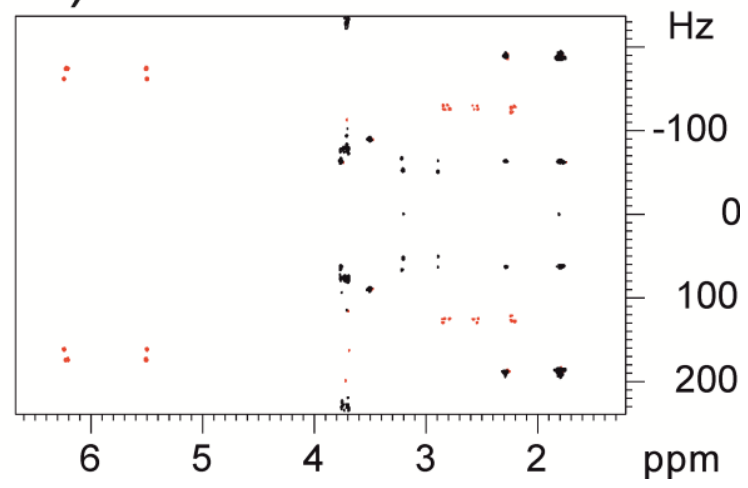

Figure S13: A-B) HD-JSB-HSQC and C-D) HD-J-HSQC spectra of ludartin (2) in isotropic $\mathrm{CDCl}_{3}$ (A and $\mathrm{C}$ ) and anisotropic $\mathrm{PMMA} \mathrm{CDCl}_{3}(\mathrm{~B}$ and $\mathrm{D})$ sample conditions. 
Table S4. Comparison of RDCs values (in $\mathrm{Hz}$ ) for ludartin (2) extracted from the corresponding HD-JSB-HSQC (Fig. S13B) and HD-J-HSQC (Fig. S13D) spectra.

\begin{tabular}{|c|c|c|}
\hline & $\begin{array}{c}{ }^{1} D_{\mathrm{CH}} \\
\text { JSB-HSQC } \\
(\mathrm{Hz})\end{array}$ & $\begin{array}{c}{ }^{1} D_{\mathrm{CH}} \text { HD- } J \text {-HSQC } \\
(\mathrm{Hz})\end{array}$ \\
\hline 2 & -6.7 & -9.1 \\
\hline 3 & -3.4 & -4.2 \\
\hline 5 & -27.8 & -27.8 \\
\hline 6 & -26.1 & -26.6 \\
\hline 7 & -24.3 & -25.4 \\
\hline 8 & -21.9 & -21.1 \\
\hline 9 & -6.7 & -10.1 \\
\hline 12 & 23.8 & 24.3 \\
\hline 14 & -1.3 & -1.7 \\
\hline 15 & -4.0 & -3.8 \\
\hline
\end{tabular}




\section{Automated extraction of ${ }^{1} J_{\mathrm{CH}}$ coupling values from $J$-resolved HSQC spectra:}

The simplified multiplet pattern feature of cross-peaks in the HD-J-HSQC spectrum offers a new approach toward an automated analysis and extraction of ${ }^{1} J_{\mathrm{CH}}$ values. An algorithm has been developed to automatically extract these ${ }^{1} J_{\mathrm{CH}}$ values thanks to the symmetry of each correlation with respect to $F_{1}=0$.

\section{Tutorial on automatic peak picking:}

1) Perform automatic peak piking analysis of both positive and negative peaks in Bruker software.

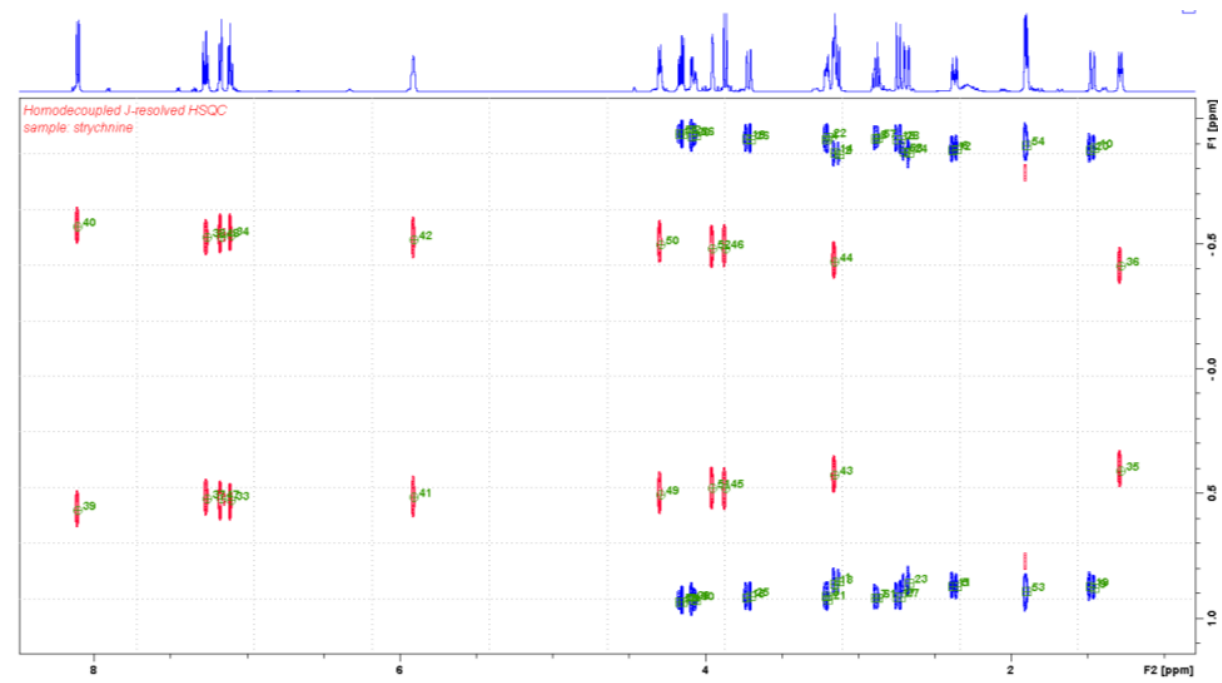

2) Order peaks in ascendant $\mathrm{F}_{2}$ frequency values (optional) and export to .csv file.

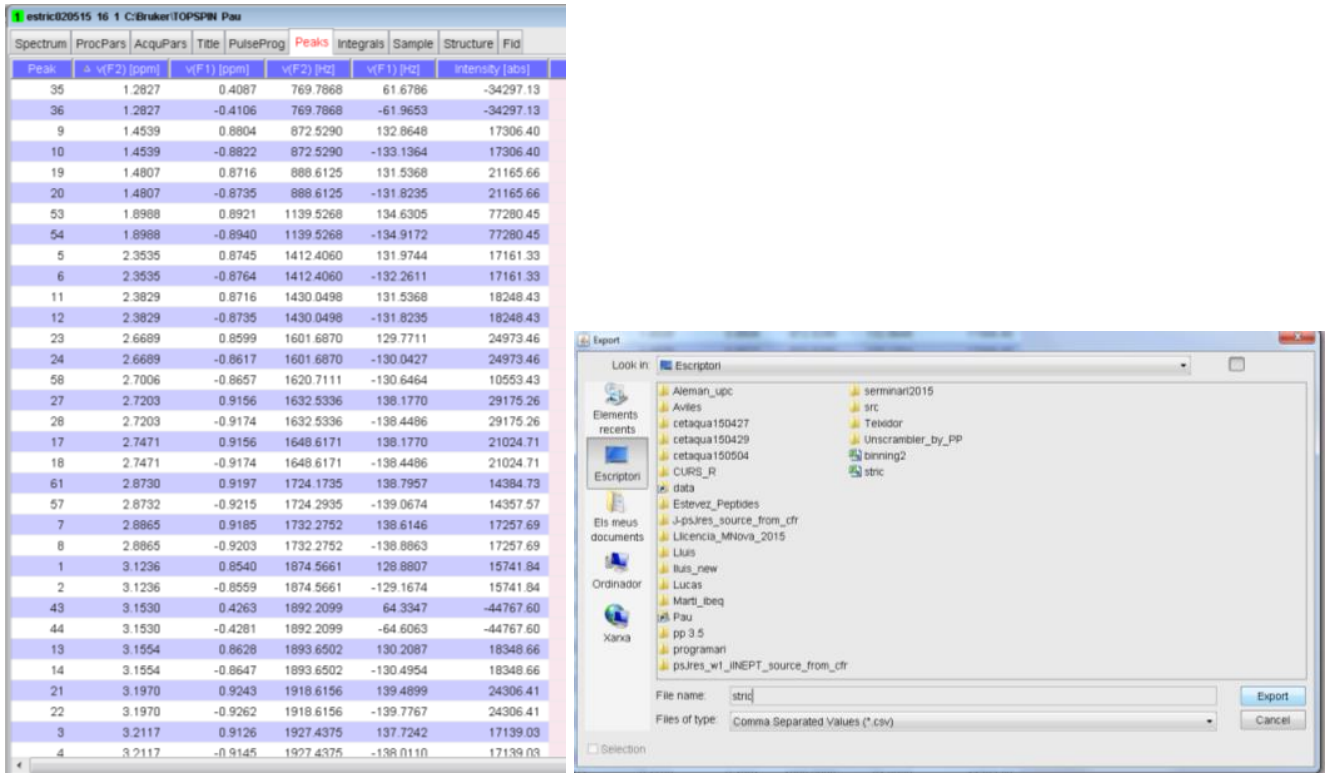


3) Open the Jmeasurement applet and browse the .csv file, click measure to perform the automatic analysis of ${ }^{1} J_{\mathrm{CH}}$. Use the print button for external printing. Use the clear button to perform a new analysis.
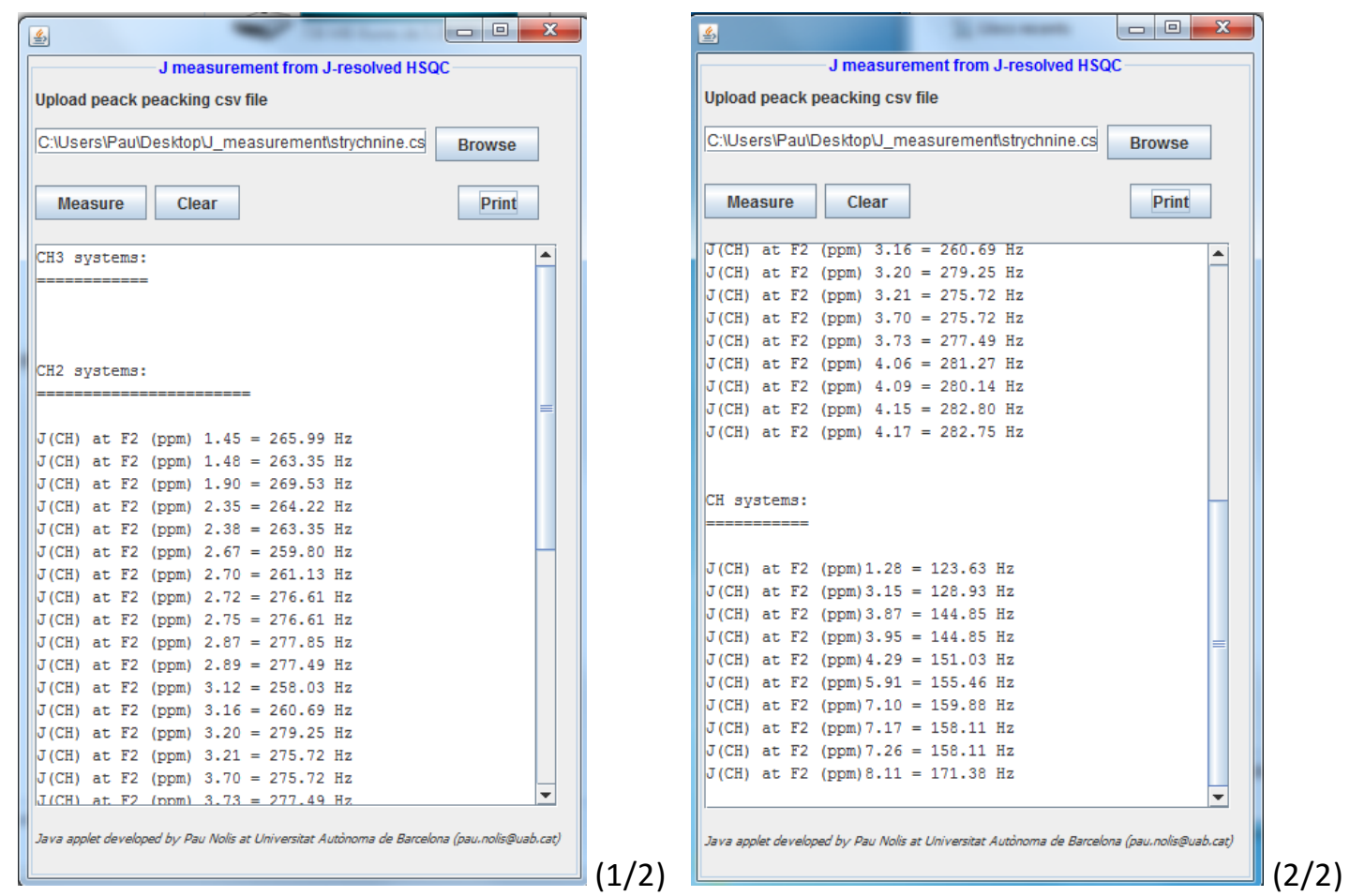

Notice that the ${ }^{1} J_{\mathrm{CH}}$ measurement is reported separately for $\mathrm{CH}_{3}, \mathrm{CH}_{2}$ and $\mathrm{CH}$ spins systems.

\section{Important note:}

In order to launch the java applet Jmeasurement.jar is a requirement to have previosuly installed in your system the JRE (Java Runtime Environtment). Any questions to pau.nolis@uab.cat. 


\section{Pulse program for Bruker spectrometers:}

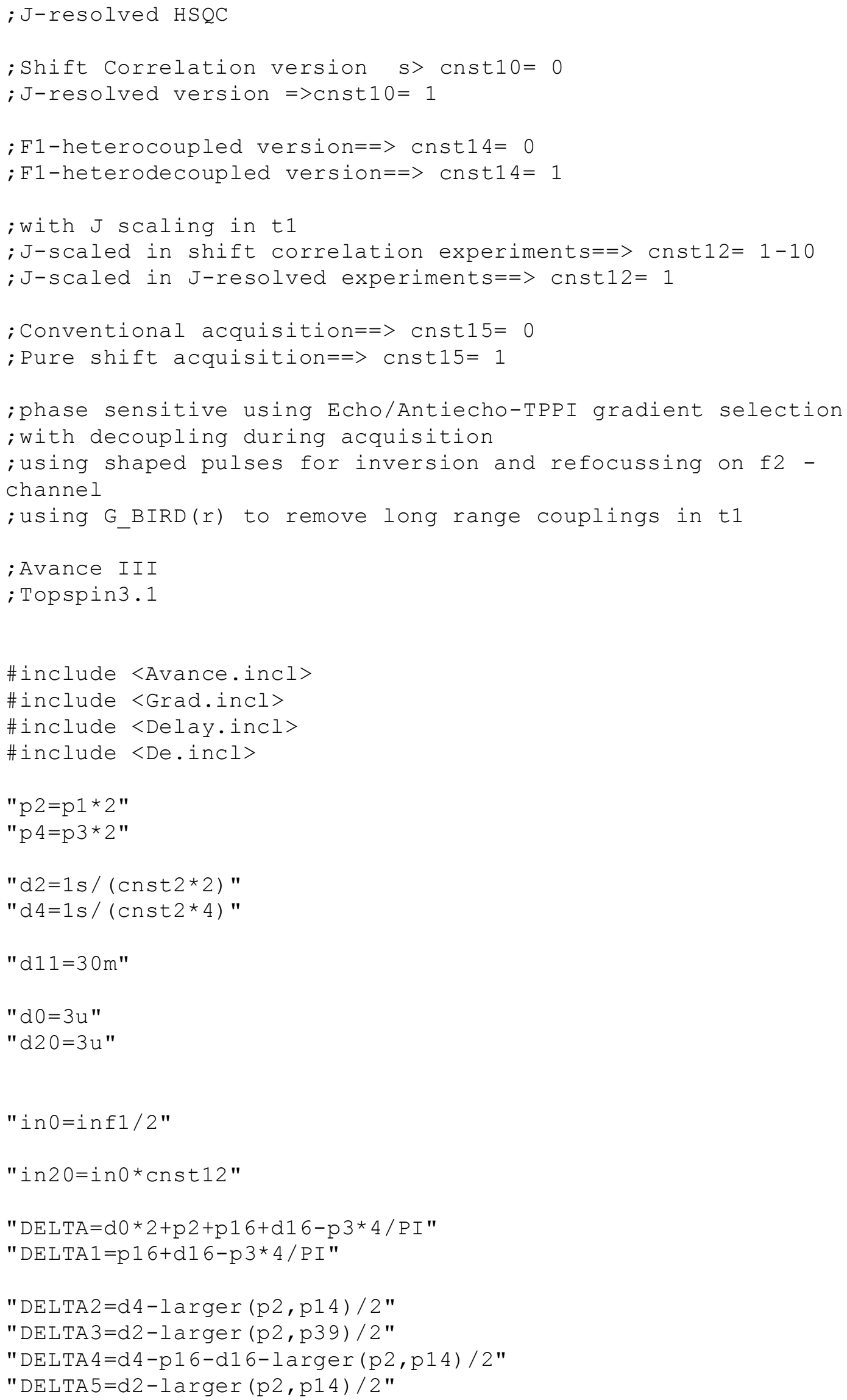


"DELTA $6=\mathrm{d} 24-\mathrm{cnst} 17 * \mathrm{p} 24 / 2-\mathrm{d} 16-4 \mathrm{u}$ "

" acqt $0=0 "$

baseopt_echo

\#define homodec

\#ifdef homodec

dwellmode explicit

"d22=aq/2 * $12 "$

\#else

\#endif

$1 \mathrm{ze}$

$2 \mathrm{~d} 11$

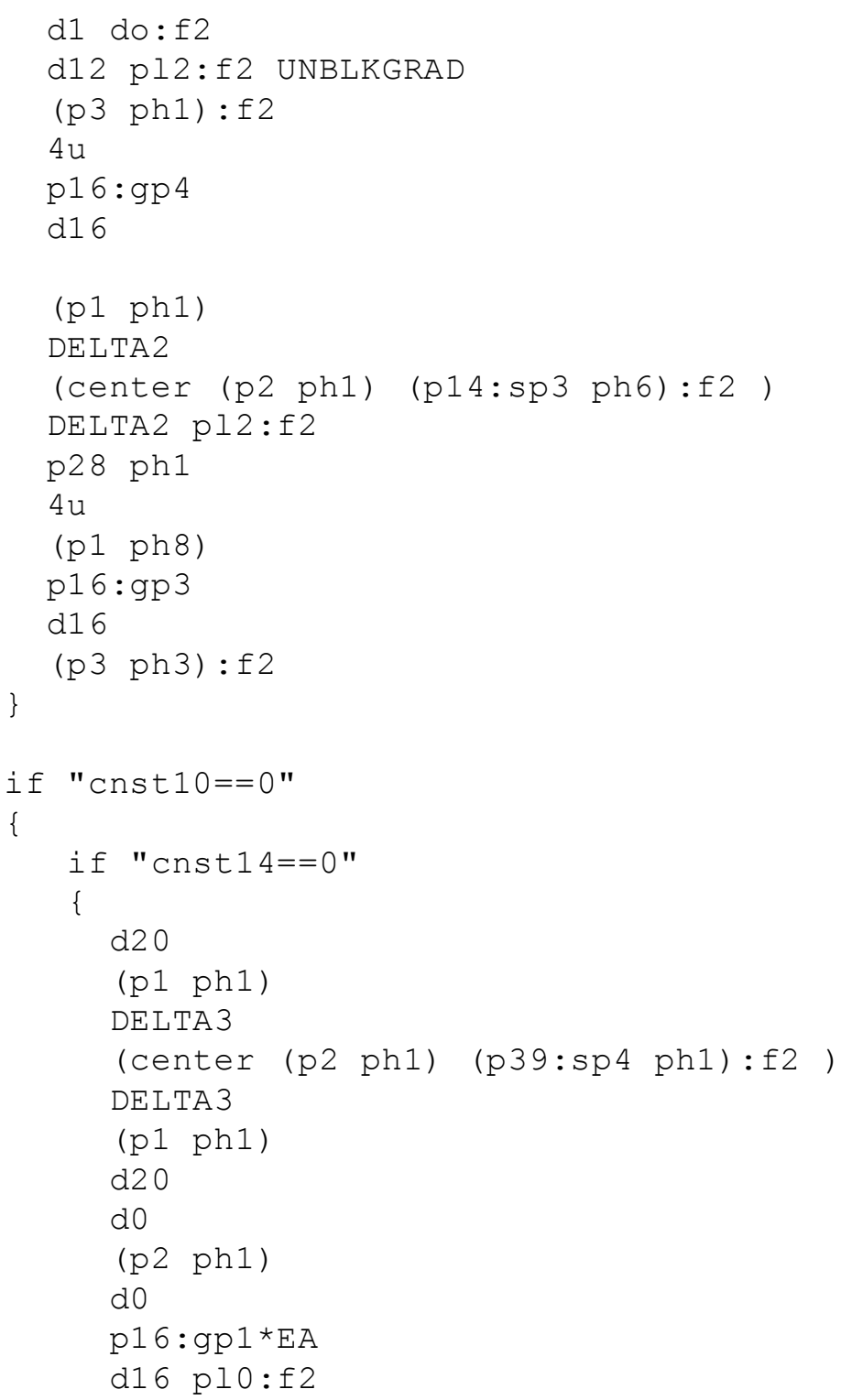




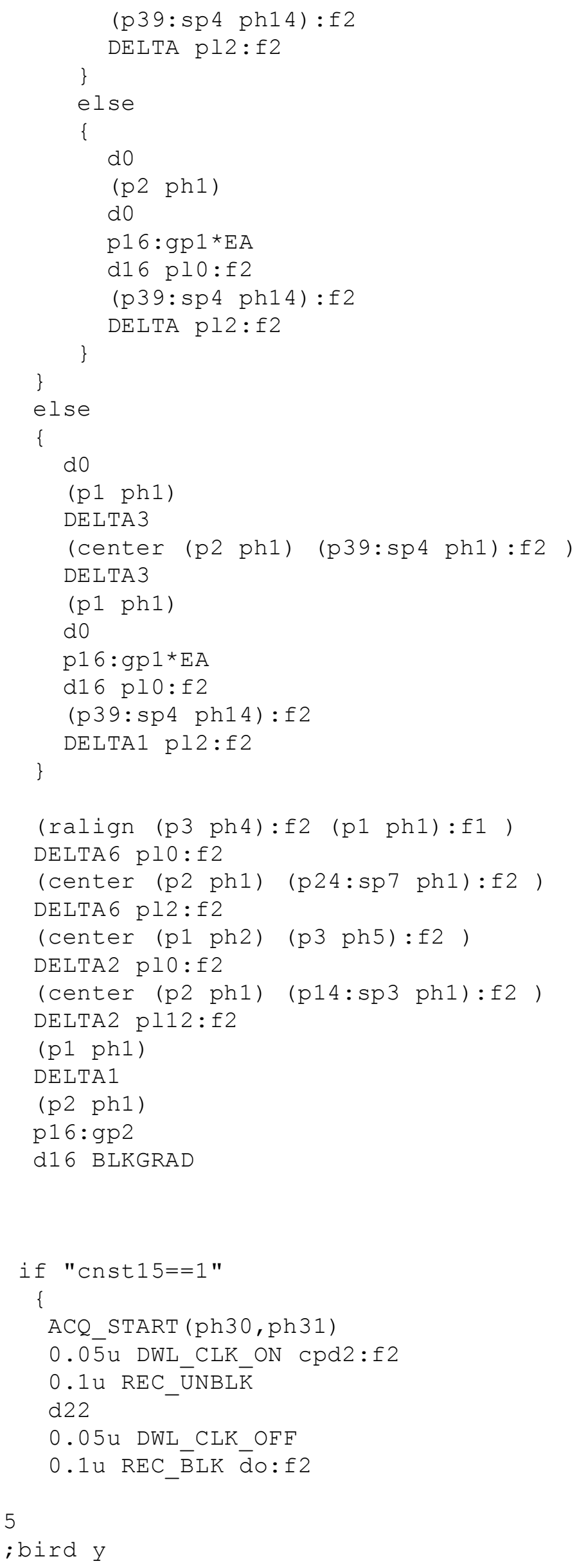




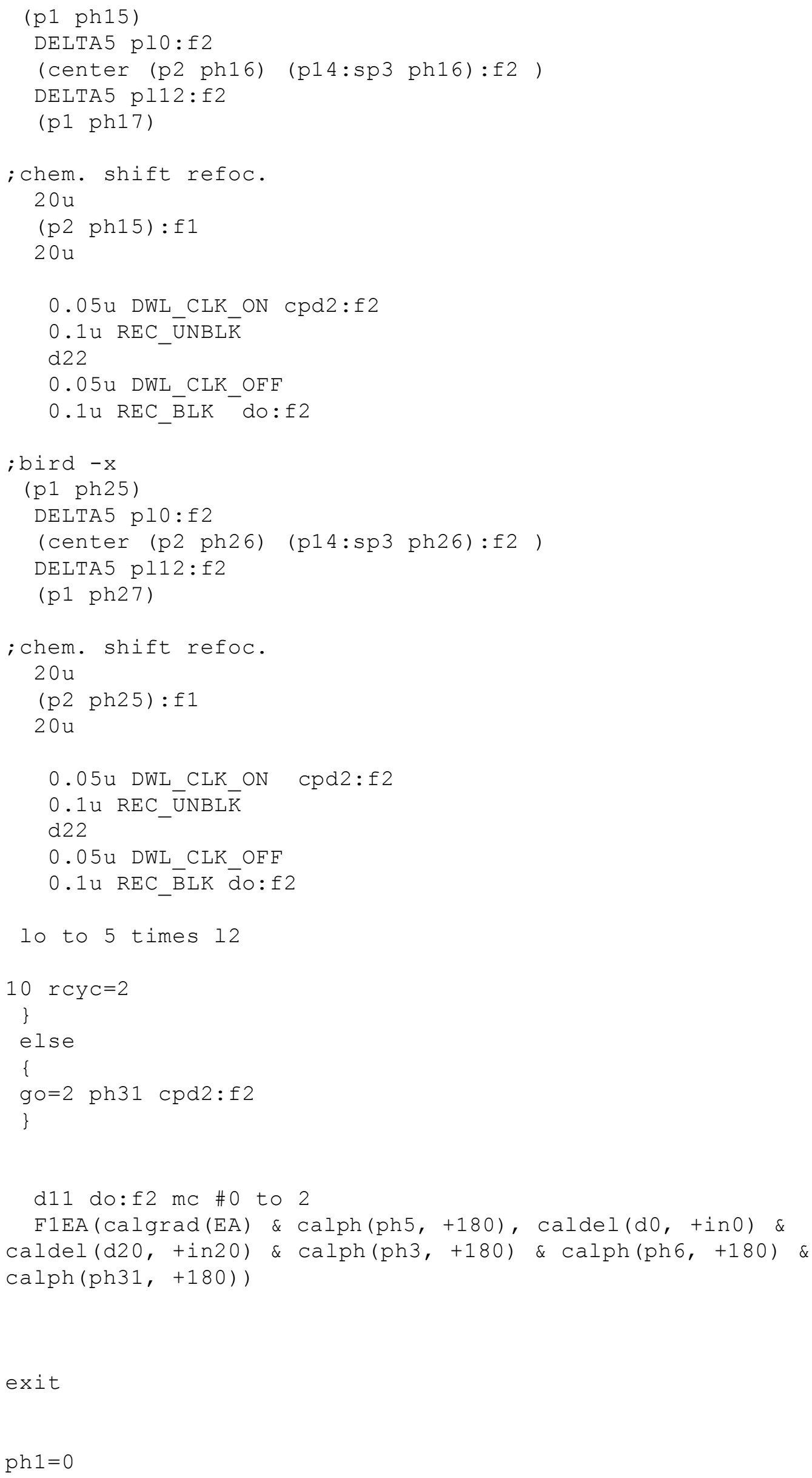

exit

$\mathrm{ph} 1=0$ 


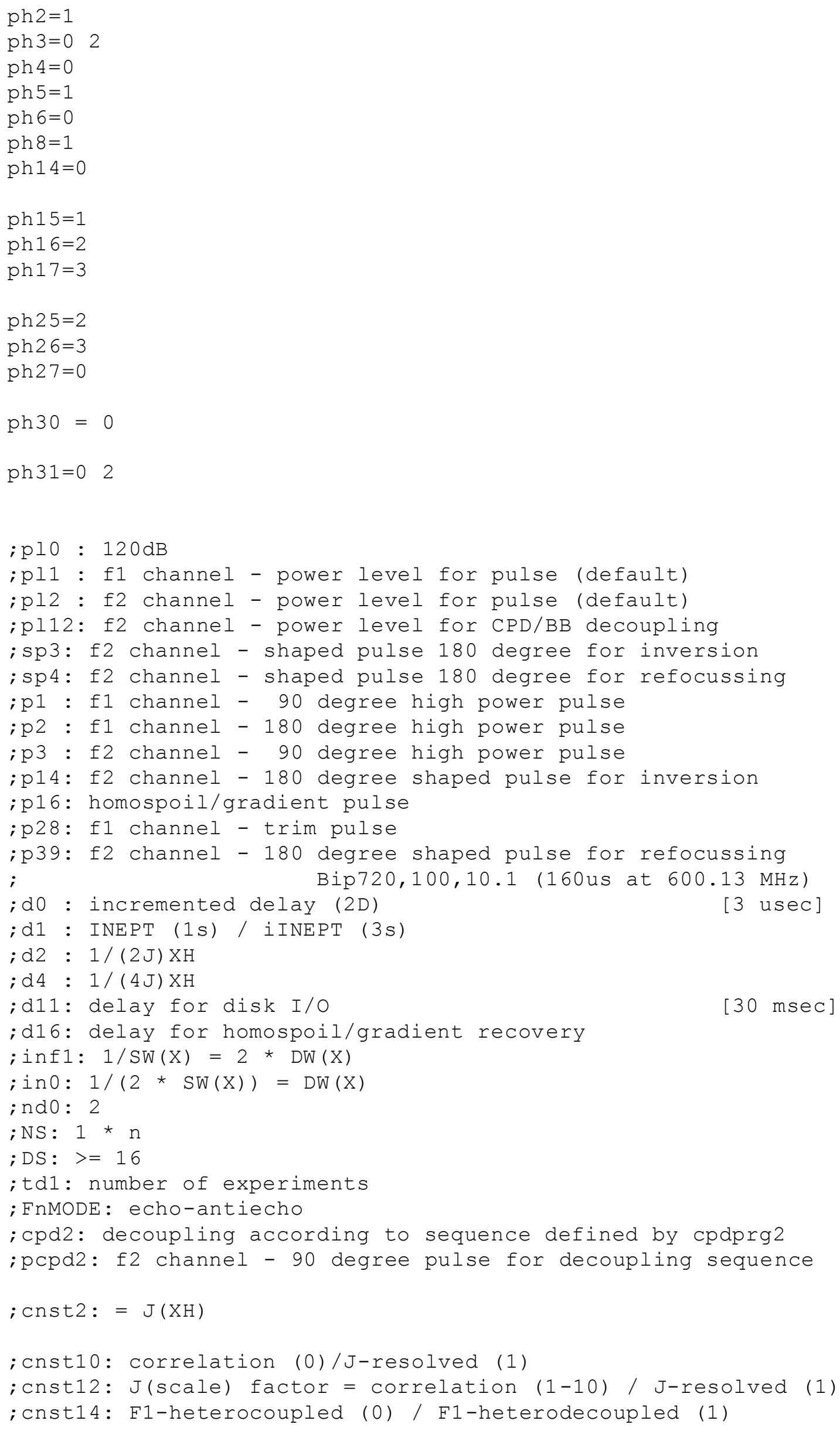


; cnst15: Conventional acquisition (0) / Pure shift acquisition (1)

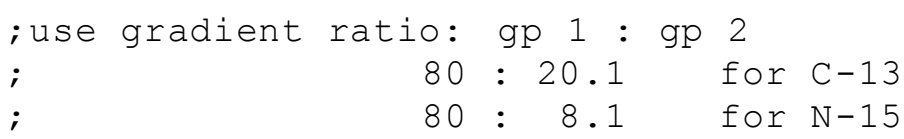

; for z-only gradients:

; gpz1: $80 \%$

; gpz2: $20.1 \%$ for $\mathrm{C}-13,8.1 \%$ for $\mathrm{N}-15$

; gpz3: 33\%

; $9 \mathrm{pz} 4: 17$ 응

; use gradient files:

igpnam1: SINE.100

; gpnam2: SINE.100

igpnam3: SINE.100

; gpnam4: SINE.100 